\title{
ON THE SECTION CONJECTURE OVER FUNCTION FIELDS AND FINITELY GENERATED FIELDS
}

\begin{abstract}
MOHAMED SAÏDI
AbStRACT. We investigate sections of arithmetic fundamental groups of hyperbolic curves over function fields. As a consequence we prove that the anabelian section conjecture of Grothendieck holds over all finitely generated fields over $\mathbb{Q}$ if it holds over all number fields, under the condition of finiteness (of the $\ell$-primary parts) of certain Shafarevich-Tate groups. We also prove that if the section conjecture holds over all number fields then it holds over all finitely generated fields for curves which are defined over a number field.
\end{abstract}

\section{Contents}

$\S 0$. Introduction

$\S 1$ Geometrically abelian fundamental groups

$\S 2$. Sections of arithmetic fundamental groups of curves over local fields of equal characteristic 0

$\S 3$. Sections of arithmetic fundamental groups of curves over function fields in characteristic 0

$\S 4$. Proofs of Theorems A and B

$\S 5$. Sections of arithmetic fundamental groups of curves over finitely generated fields in characteristic 0

123

$\S \mathbf{0}$. Introduction. Let $k$ be a characteristic 0 field and $X$ a smooth, projective, and geometrically connected hyperbolic curve (i.e., $g(X) \geq 2$ ) over $k$. Let $\pi_{1}(X)$ be the arithmetic étale fundamental group of $X$ which sits in the following exact sequence

$$
1 \rightarrow \pi_{1}(\bar{X}) \rightarrow \pi_{1}(X) \stackrel{\text { pr }}{\longrightarrow} G_{k} \stackrel{\text { def }}{=} \operatorname{Gal}(\bar{k} / k) \rightarrow 1,
$$

where $\bar{k}$ is an algebraic closure of $k$ and $\bar{X}=X \times_{k} \bar{k}$. In this paper we investigate continuous group-theoretic sections (i.e., splittings) $s: G_{k} \rightarrow \pi_{1}(X)$ of the projection pr: $\pi_{1}(X) \rightarrow G_{k}$, which we will refer to as sections of $\pi_{1}(X)$.

Sections of $\pi_{1}(X)$ arise naturally from $k$-rational points of $X$. More precisely, a rational point $x \in X(k)$ determines a decomposition subgroup $D_{x} \subset \pi_{1}(X)$, which is defined modulo conjugation by the elements of $\pi_{1}(\bar{X})$, and which maps isomorphically to $G_{k}$ via the projection pr : $\pi_{1}(X) \rightarrow G_{k}$. (Thus, $D_{x}$ determines a

\footnotetext{
${ }^{1}$ Communicated by S. Mochizuki. Received January 14, 2016. Revised March 14, 2016.

${ }^{2} 2010$ Mathematics Subject Classification: 11G30, 14H25, 14H30, 14 G05.

${ }^{3}$ Exeter university, e-mail address: M.Saidi@exeter.ac.uk
} 
splitting of the above exact sequence.) We will refer to such a section of $\pi_{1}(X)$ as point-theoretic, and say that it arises from the rational point $x \in X(k)$. We have a set-theoretic map

$$
\varphi_{X}: X(k) \rightarrow \overline{\operatorname{Sec}}_{\pi_{1}(X)}, \quad x \mapsto \varphi_{X}(x)=\left[s_{x}\right]
$$

where $\overline{\operatorname{Sec}}_{\pi_{1}(X)}$ is the set of conjugacy classes of sections of $\pi_{1}(X)$, modulo conjugation by the elements of $\pi_{1}(\bar{X})$, and $\left[s_{x}\right]$ denotes the image (i.e., conjugacy class) of a section $s_{x}$ associated to $x \in X(k)$.

Definition 0.1. (i) We say that the SC (section conjecture) holds for $X$, over $k$, if the above map $\varphi_{X}: X(k) \rightarrow \overline{\operatorname{Sec}}_{\pi_{1}(X)}$ is bijective.

(ii) We say that the SC holds over $k$ if the $\mathbf{S C}$ holds for every smooth, projective, and geometrically connected hyperbolic curve $X$ over $k$ (cf. (i)).

In his seminal letter to Faltings, Grothendieck formulated the following conjecture (cf. [Grothendieck]).

Grothendieck's Anabelian Section Conjecture (GASC). Assume that $k$ is finitely generated over the prime field $\mathbb{Q}$. Then the $\mathbf{S C}$ holds over $k$.

The injectivity of the map $\varphi_{X}$ if $k$ is finitely generated over $\mathbb{Q}$, or more generally if $k$ is a sub- $p$-adic field, is well-known (cf. [Mochizuki], Theorem C). The statement of the GASC is thus equivalent to the surjectivity of the map $\varphi_{X}$, i.e., that every section of $\pi_{1}(X)$ is point-theoretic under the above assumptions on the field $k$. The GASC, even over number fields, is still wide open. More generally, one can ask: what are all fields (of characteristic 0) for which the $\mathbf{S C}$ holds? In this paper we investigate the section conjecture over function fields of curves in characteristic 0.

Given a separated, smooth, and connected curve $C$ over $k$ with function field $K \stackrel{\text { def }}{=} k(C)$, an abelian scheme $\mathcal{A} \rightarrow C$ with generic fibre $A \stackrel{\text { def }}{=} \mathcal{A} \times{ }_{C}$ Spec $K$, define the Shafarevich-Tate group

$$
\amalg(\mathcal{A})=\amalg(A, C) \stackrel{\text { def }}{=} \operatorname{Ker}\left(H^{1}\left(G_{K}, A\right) \rightarrow \prod_{c \in C^{\mathrm{cl}}} H^{1}\left(G_{K_{c}}, A_{c}\right)\right),
$$

where $c \in C^{\mathrm{cl}}$ is a closed point, $K_{c}$ is the completion of $K$ at $c, A_{c} \stackrel{\text { def }}{=} A \times_{\text {Spec } K}$ Spec $K_{c}$, and the product is over all closed points of $C$.

Definition 0.2. Let $k^{\prime}$ be a field with $\operatorname{char}\left(k^{\prime}\right)=0$. Consider the following conditions.

(i) The $\ell^{\prime}$-cyclotomic character $\chi_{\ell^{\prime}}: G_{k^{\prime}} \rightarrow \mathbb{Z}_{\ell^{\prime}}^{\times}$is non-Tate in the sense of [CadoretTamagawa] (§2, Definition), meaning that the 1 dimensional $\ell^{\prime}$-adic representation $\mathbb{Z}_{\ell^{\prime}}(1)$ doesn't appear as a sub-representation of the representation arising from the $\ell^{\prime}$-adic Tate module of an abelian variety over $k^{\prime} ; \forall$ prime integer $\ell^{\prime}$.

(ii) The SC holds over $k^{\prime}$.

(iii) Given a separated, smooth, and connected curve $C$ over $k^{\prime}$, with function field $K \stackrel{\text { def }}{=} k^{\prime}(C)$, an abelian scheme $\mathcal{A} \rightarrow C$, then $T \amalg(\mathcal{A})=0$ (cf. Notations).

(iv) Given an abelian variety $A$ over $k^{\prime}$, the group of $k^{\prime}$-rational points $A\left(k^{\prime}\right)$, and a quotient $A\left(k^{\prime}\right) \rightarrow D$, the following hold.

(a) The natural map $D \rightarrow D^{\wedge}$ (cf. Notations) is injective.

(b) The torsion group $D[N]$ is finite $\forall N \geq 1$, and $T D=0$ (cf. Notations). 
(v) Given a function field $K=k^{\prime}(C)$ as in (iii), $K$ admits a structure of Hausdorff topological field so that $X(K)$ becomes compact for any proper, smooth, and geometrically connected hyperbolic curve over $K$.

(vi) Given a separated, smooth, and connected curve $C$ over $k^{\prime}$ with function field $K \stackrel{\text { def }}{=} k^{\prime}(C)$, a finite étale morphism $\widetilde{C} \rightarrow C$, then the following hold. If $\widetilde{C}_{c}\left(k^{\prime}(c)\right) \neq \emptyset, \forall c \in C^{\mathrm{cl}}$ with residue field $k^{\prime}(c)$ and where $\widetilde{C}_{c}$ is the scheme-theoretic inverse image of $c$ in $\widetilde{C}$, then $\widetilde{C}(K) \neq \emptyset$.

Given a field $k$ with $\operatorname{char}(k)=0$, we say that $k$ strongly satisfies one of the conditions (i), (ii), (iii), (iv), (v), and (vi) above, if this condition is satisfied by any finite extension $k^{\prime} / k$ of $k$. We say that the field $k$ satisfies the condition $(\star)$ if $k$ strongly satisfies each of the conditions (i), (ii), (iii), (iv), (v), and (vi).

Conditions (i), (iv), (v), and (vi) above are satisfied by finitely generated fields over $\mathbb{Q}$. In this case, (i) follows from the theory of weights, (iv) follows from the Mordell-Weil and Lang-Néron Theorems, (v) follows (for the discrete topology) from Mordell's conjecture: Faltings' Theorem and Néron's specialisation Theorem, and (vi) follows from the Hilbertian property (cf. Lemma 4.1.5) which holds for finitely generated fields. It is not known in general if condition (iii) holds over such fields (cf. [Saïdi-Tamagawa] for some partial results).

One of our main results in this paper are the following.

Theorem A. Let $k$ be a field with $\operatorname{char}(k)=0$ and $C$ a separated, smooth, and connected curve over $k$ with function field $K=k(C)$. Assume that $k$ strongly satisfies the conditions (i), (ii), (iv), (v), and (vi) in Definition 0.2. Let $\mathcal{X} \rightarrow C$ be a flat, proper, and smooth relative curve, with generic fibre $X \stackrel{\text { def }}{=} \mathcal{X} \times_{C} \operatorname{Spec} K$ which is a geometrically connected hyperbolic curve over $K$, and $\mathcal{J} \stackrel{\text { def }}{=} \operatorname{Pic}_{\mathcal{X} / C}^{0}$ its relative jacobian. Assume that $X(K) \neq \emptyset$, and $T \amalg(\mathcal{J})=0$. Then the $\mathbf{S C}$ holds for $X$ (over $K$ ).

Theorem B. Let $k$ be a field with $\operatorname{char}(k)=0$, and $K=k(C)$ the function field of a separated, smooth, and connected curve $C$ over $k$. Assume that $k$ satisfies the condition ( $\star$ ) (cf. Definition 0.2). Then $K$ strongly satisfies the condition (ii). Equivalently, if $L / K$ is a finite extension then the $\mathbf{S C}$ holds over $L$.

In the case of finitely generated fields one obtains immediately from Theorem A, and Theorem B; respectively, the following Corollaries.

Corollary A. Assume that the $\mathbf{S C}$ holds over all finitely generated fields over $\mathbb{Q}$ of transcendence degree $i \geq 0$. Let $k$ be a field with $\operatorname{tr} \operatorname{deg}_{\mathbb{Q}} k=i$ and $C$ a separated, smooth, and connected curve over $k$ with function field $K=k(C)$. Let $\mathcal{X} \rightarrow C$ be a flat, proper, and smooth relative curve, with generic fibre $X \stackrel{\text { def }}{=} \mathcal{X} \times_{C}$ Spec $K$ which is a geometrically connected hyperbolic curve over $K$, and $\mathcal{J} \stackrel{\text { def }}{=} \mathrm{Pic}_{\mathcal{X} / C}^{0}$ its relative jacobian. Assume that $X(K) \neq \emptyset$, and $T \amalg(\mathcal{J})=0$. Then the $\mathbf{S C}$ holds for $X$ (over $K$ ).

Corollary B. Assume that the SC holds over all number fields (i.e., all finite extensions of $\mathbb{Q}$ ) and that the condition (iii) (in Definition 0.2) holds for any field $k^{\prime}$ which is finitely generated over $\mathbb{Q}$. Then the $\mathbf{S C}$ holds over all finitely generated fields over $\mathbb{Q}$. 
Recently, with Akio Tamagawa, we proved that the Shafarevich-Tate group $\amalg(\mathcal{J})$ in Theorem $\mathrm{A}$ is indeed finite if the jacobian $J \stackrel{\text { def }}{=} \operatorname{Pic}_{X / K}^{0}$ of $X$ is (isogenous to) a constant abelian variety, i.e., with the notations in Definition 0.2 the condition (iii) holds for abelian schemes $\mathcal{A} \rightarrow C$ such that $A \stackrel{\text { def }}{=} \mathcal{A} \times{ }_{C} \operatorname{Spec} K$ (is isogenous to an abelian variety which) descends (over $\bar{K}$ ) to an abelian variety defined over a finite extension of $k^{\prime}$ (cf. [Saïdi-Tamagawa], Theorem H). As a consequence of this result, and Theorem A, one deduces (using an induction argument) the following (cf. Corollary 5.3).

Theorem C. Let $K$ be a finitely generated field over $\mathbb{Q}$ with algebraic closure $\bar{K}$ and $\overline{\mathbb{Q}}$ the algebraic closure of $\mathbb{Q}$ in $\bar{K}$. Assume that the $\mathbf{S C}$ holds over all number fields. Then the $\mathbf{S C}$ holds for every projective, smooth, and geometrically connected hyperbolic curve $X$ over $K$ such that $X \times_{\operatorname{Spec} K} \operatorname{Spec} \bar{K}$ is defined over $\overline{\mathbb{Q}}$.

Our method to prove Theorem A relies on a local-global approach and follows from a thorough investigation of sections of arithmetic fundamental groups of hyperbolic curves over local fields of equal characteristic 0, and over function fields of curves in characteristic 0. Next, we explain the content of each section. In $\S 1$ we establish some basic facts on geometrically abelian fundamental groups and their sections. In $\S 2$ we investigate (under the assumption that condition (i) holds) sections of arithmetic fundamental groups of curves over local fields of equal characteristic 0 . We observe that the section conjecture SC doesn't hold over local fields of equal characteristic 0 (cf. Lemma 2.1.3 and Proposition 2.3.1). We discuss those sections which are point-theoretic in the case of stable curves (cf. Lemma 2.1.4 and Lemma 2.2.2). In $\S 3$ we investigate sections of arithmetic fundamental groups of curves over function fields (of transcendence degree 1), and establish some of the basic techniques and facts in order to investigate their point-theoreticity via a local-global approach. In $\S 4$ we prove Theorem A and explain how Theorem B can be derived from Theorem A. Finally, in $\S 5$ we relate the results and techniques of $\S 3$, and $\S 4$, to the results and framework in [Saïdi-Tamagawa]. As a consequence, we prove some variants of Theorems A and B involving a condition of finiteness of the $\ell$-primary parts of certain Shafarevich-Tate groups (cf. Corollary 5.2 and Theorem 5.4), and deduce Theorem C.

Theorems A, B, and C concern sections of arithmetic fundamental groups of proper curves over function fields and finitely generated fields. One can prove similar results for non-cuspidal sections of arithmetic fundamental groups of affine curves over function fields and finitely generated field, as well as for the birational version of the section conjecture (cf. [Saïdi2]).

Acknowledgment. I would like to thank Akio Tamagawa for several discussions we had on the topic of this paper, as well as for his help with some technical details. Part of this work was done while the author was visiting the Research Institute of Mathematical Sciences (RIMS) of Kyoto university. I would like very much to thank the members of the Institute for their hospitality.

Notations. Given a scheme $Y$ over a field $L$ with algebraic closure $\bar{L}$ we write $Y_{\bar{L}} \stackrel{\text { def }}{=} Y \times_{\text {Spec } L}$ Spec $\bar{L}$ for the geometric fibre of $Y$. Given a scheme $C$, a field $L$, $Y \rightarrow C$ and $\operatorname{Spec} L \rightarrow C$ morphisms of schemes, we write $Y_{L} \stackrel{\text { def }}{=} Y \times_{C} \operatorname{Spec} L$. For an algebraic group $G$ over a field $L$ of characteristic 0 , with algebraic closure $\bar{L}$, we 
write $T G \stackrel{\text { def }}{=} \varliminf_{N \geq 1} G[N](\bar{L})$ for the Tate module of $G$, where $G[N] \stackrel{\text { def }}{=} \operatorname{Ker}(G \stackrel{[N]}{\longrightarrow} G)$ is the kernel of the homomorphism of multiplication by $N$. For a profinite group $H$ we write $H^{\mathrm{ab}}$ for the maximal abelian quotient of $H$. For an abelian group $D$ we write $D^{\wedge} \stackrel{\text { def }}{=} \underbrace{}_{N \geq 1} D / N D$, where $N D \stackrel{\text { def }}{=}\{N . a \mid a \in D\}$. Given an integer $N \geq 1$, we

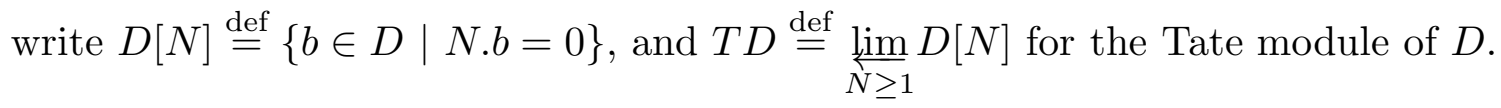

$\S 1$ Geometrically abelian fundamental groups. Let $K$ be a field of characteristic $0, X \rightarrow$ Spec $K$ a proper, smooth, and geometrically connected hyperbolic curve over $K$. Let $\xi$ be a geometric point of $X$ with value in its generic point. Thus, $\xi$ determines an algebraic closure $\bar{K}$ of $K$. Write $\bar{\xi}$ for the geometric point of $X_{\bar{K}}$ which is induced by $\xi$. We have an exact sequence of étale fundamental groups

$$
1 \rightarrow \pi_{1}\left(X_{\bar{K}}, \bar{\xi}\right) \rightarrow \pi_{1}(X, \xi) \stackrel{\mathrm{pr}}{\longrightarrow} G_{K} \rightarrow 1
$$

where $G_{K} \stackrel{\text { def }}{=} \operatorname{Gal}(\bar{K} / K)$. Write

$$
\pi_{1}(X, \xi)^{(\mathrm{ab})} \stackrel{\text { def }}{=} \pi_{1}(X, \xi) / \operatorname{Ker}\left(\pi_{1}\left(X_{\bar{K}}, \bar{\xi}\right) \rightarrow \pi_{1}\left(X_{\bar{K}}, \bar{\xi}\right)^{\mathrm{ab}}\right)
$$

We will refer to $\pi_{1}(X, \xi)^{(\mathrm{ab})}$ as the geometrically abelian quotient of $\pi_{1}(X, \xi)$.

Assume that $X(K) \neq \emptyset$. Write $J \stackrel{\text { def }}{=} \operatorname{Pic}_{X / K}^{0}$ for the jacobian variety of $X$, and $\iota: X \rightarrow J$ for the embedding which maps a rational point $x_{0} \in X(K)$ to the zero section of $J$. Then $\iota$ induces a commutative diagram of exact sequences

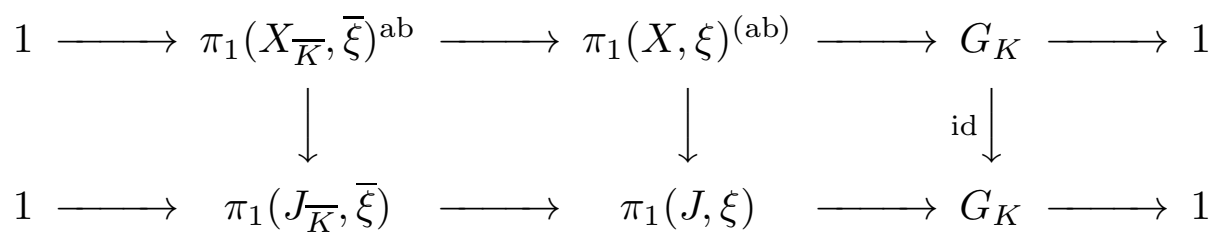

where the vertical maps are isomorphisms, hence an identification of $G_{K}$-modules $\pi_{1}\left(X_{\bar{K}}, \bar{\xi}\right)^{\mathrm{ab}} \stackrel{\sim}{\rightarrow} \pi_{1}\left(J_{\bar{K}}, \bar{\xi}\right) \stackrel{\sim}{\rightarrow} T J$. Let

$$
s: G_{K} \rightarrow \pi_{1}(X, \xi)
$$

be a section of $\pi_{1}(X, \xi)$. Then $s$ induces a section

$$
s^{\mathrm{ab}}: G_{K} \rightarrow \pi_{1}(X, \xi)^{(\mathrm{ab})}
$$

of the projection $\pi_{1}(X, \xi)^{(\mathrm{ab})} \rightarrow G_{K}$. The set of splittings of the upper sequence in diagram (1.2) is, up to conjugation by the elements of $\pi_{1}\left(X_{\bar{K}}, \bar{\xi}\right)^{\mathrm{ab}}$, a torsor under the Galois cohomology group $H^{1}\left(G_{K}, \pi_{1}\left(X_{\bar{K}}, \bar{\xi}\right)^{\mathrm{ab}}\right) \stackrel{\sim}{\rightarrow} H^{1}\left(G_{K}, T J\right)$. We fix a base point of the torsor of splittings of this exact sequence to be the splitting arising from the zero section of $J$, i.e., the splitting arising from the rational point $x_{0} \in X(K)$ (cf. above discussion). Then the above (conjugacy class of the) section $s^{\mathrm{ab}}$ corresponds to an element

$$
s^{\mathrm{ab}} \in H^{1}\left(G_{K}, T J\right) .
$$


We will refer to $s^{\mathrm{ab}}$ as the abelian portion of the section $s$.

The Kummer exact sequences $0 \rightarrow J[N] \rightarrow J \stackrel{N}{\rightarrow} J \rightarrow 0, \forall N \geq 1$, induce an exact sequence; the so-called Kummer exact sequence

$$
0 \rightarrow J(K)^{\wedge} \rightarrow H^{1}\left(G_{K}, T J\right) \rightarrow T H^{1}\left(G_{K}, J\right) \rightarrow 0
$$

We will identify $J(K)^{\wedge}$ with its image in $H^{1}\left(G_{K}, T J\right)$ via the above Kummer map $J(K)^{\wedge} \rightarrow H^{1}\left(G_{K}, T J\right)$. Note that there exist natural maps $X(K) \stackrel{\iota}{\rightarrow} J(K) \rightarrow$ $J(K)^{\wedge}$, where for $x \in X(K)$ the image $\iota(x)$ is the class $\left[x-x_{0}\right]$ of the degree 0 divisor $x-x_{0}$.

Definition 1.1. Let $\eta \in H^{1}\left(G_{K}, T J\right)$.

(i) We say that $\eta$ is pro-geometric if $\eta$ lies in the subgroup $J(K)^{\wedge}$ of $H^{1}\left(G_{K}, T J\right)$.

(ii) We say that $\eta$ is geometric if $\eta$ is in the image of the natural composite homomorphism $J(K) \rightarrow J(K)^{\wedge} \rightarrow H^{1}\left(G_{K}, T J\right)$.

(iii) We say that $\eta$ is point-theoretic if $\eta$ is in the image of the composite map $X(K) \stackrel{\iota}{\rightarrow} J(K) \rightarrow J(K)^{\wedge} \rightarrow H^{1}\left(G_{K}, T J\right)$.

The following Lemma follows easily from the various Definitions.

Lemma 1.2. Suppose that the section $s=s_{x}, x \in X(K)$, is point-theoretic (cf. $\S 0)$. Then $s^{\mathrm{ab}} \in H^{1}\left(G_{K}, T J\right)$ is point-theoretic (cf. Definition 1.1(iii)), and $s^{\mathrm{ab}}$ is the image of $x$ via the composite map $X(K) \stackrel{\iota}{\rightarrow} J(K) \rightarrow J(K)^{\wedge} \rightarrow H^{1}\left(G_{K}, T J\right)$. In particular, $s^{\mathrm{ab}}$ is pro-geometric and geometric (cf. Definition 1.1(i) (ii)).

$\S 2$. Sections of arithmetic fundamental groups of curves over local fields of equal characteristic 0 . In this section $K$ is a complete discrete valuation field of equal characteristics $0, \mathcal{O}_{K}$ its valuation ring, and $k$ its residue field. We use the notations introduced in $\S 0$ and $\S 1$. Moreover, we assume that $k$ satisfies the condition (i) in Definition 0.2, unless we specify otherwise.

Let $X \rightarrow \operatorname{Spec} \mathcal{O}_{K}$ be a flat, proper, stable, and geometrically connected (relative) curve over $\mathcal{O}_{K}$, with $X_{K}$ smooth. Assume that the irreducible components $\left\{X_{i}\right\}_{i \in I}$ of $X_{k}=\sum_{i \in I} X_{i}$ are smooth, geometrically connected, and the singular points $\left\{x_{j}\right\}_{j \in J}$ of $X_{k}$ are $k$-rational. Let $\xi$ (resp. $\xi^{\prime}$ ) be a geometric point of $X$ with value in the generic point of $X$ (resp. with value in the generic point of some irreducible component $X_{i_{0}}$ of $X_{k}$ ). Thus, $\xi$ (resp. $\xi^{\prime}$ ) determines an algebraic closure $\bar{K}$ (resp. $\bar{k}$ ) of $K$ (resp. $k$ ). We have natural exact sequences of arithmetic "admissible"fundamental groups

$$
1 \rightarrow \pi_{1}\left(X_{\bar{K}}, \bar{\xi}\right) \rightarrow \pi_{1}\left(X_{K}, \xi\right) \rightarrow G_{K} \rightarrow 1
$$

and

$$
1 \rightarrow \pi_{1}\left(X_{\bar{k}}, \bar{\xi}^{\prime}\right)^{\mathrm{adm}} \rightarrow \pi_{1}\left(X_{k}, \xi^{\prime}\right)^{\mathrm{adm}} \rightarrow G_{K} \rightarrow 1
$$

where $G_{K} \stackrel{\text { def }}{=} \operatorname{Gal}(\bar{K} / K)$, and the geometric point $\bar{\xi}$ (resp. $\bar{\xi}^{\prime}$ ) is naturally induced by $\xi$ (resp. $\left.\xi^{\prime}\right)$. Here, the superscript "adm" means admissible fundamental group (cf. [Mochizuki1], Definition 2.7, for more details on the definition of $\pi_{1}\left(X_{\bar{k}}, \bar{\xi}^{\prime}\right)^{\text {adm }}$. We also refer to [Mochizuki1], the discussion before Definition 2.1, as well as the discussion between Definition 2.5 and Definition 2.7, for the precise definition of the $\log$ structures on $X_{k}$, Spec $k, \operatorname{Spec} \mathcal{O}_{K}, X_{k} \rightarrow \operatorname{Spec} k$, and $X \rightarrow \operatorname{Spec} \mathcal{O}_{X}$ involved 
in the definition of $\left.\pi_{1}\left(X_{\bar{k}}, \bar{\xi}^{\prime}\right)^{\text {adm }}\right)$. Moreover, after a suitable choice of $\xi$ and $\xi^{\prime}$ we have a commutative diagram of exact sequences

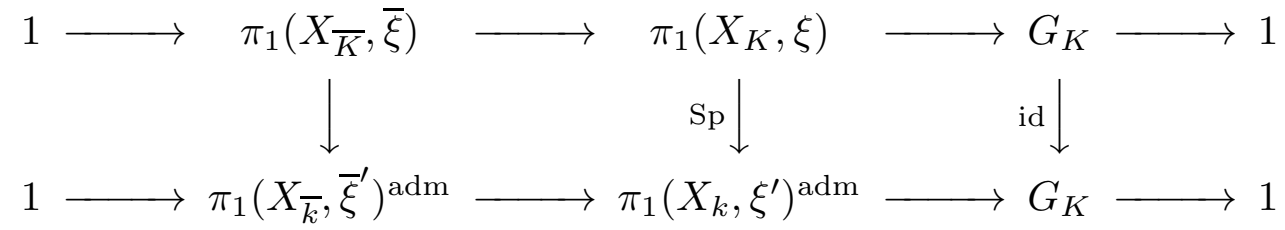

where the middle and left vertical maps are continuous homomorphisms of "specialisation", which are isomorphisms since $\operatorname{char}(k)=0$ (cf. loc. cit. Definition 2.4, Lemma 2.6, and the discussion before Definition 2.7). We assume that $X_{K}$ is hyperbolic, i.e., $g\left(X_{K}\right) \geq 2$. We have an exact sequence $1 \rightarrow I_{K} \rightarrow G_{K} \rightarrow G_{k} \stackrel{\text { def }}{=}$ $\operatorname{Gal}(\bar{k} / k) \rightarrow 1$ where $I_{K}$ is the inertia group. Moreover, $I_{K} \stackrel{\sim}{\rightarrow} \hat{\mathbb{Z}}(1)$ where the "(1)" is a Tate twist.

2.1. The good reduction case. Assume that $X$ is smooth. In this case we have a commutative diagram of exact sequences of arithmetic fundamental groups

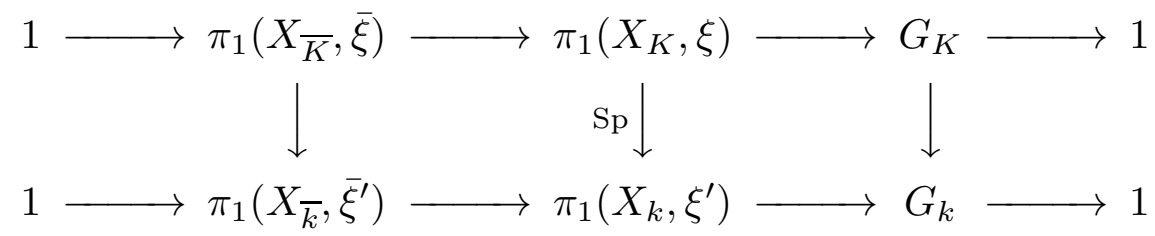

where the middle vertical map is the surjective homomorphism of specialisation (defined up to conjugation, cf. [Grothendieck1], Exposé X, §2), the left vertical map is an isomorphism ( $\operatorname{since} \operatorname{char}(k)=0$ ), and the right vertical map is a surjection.

Lemma 2.1.1. The following hold.

(i) The right square in diagram (2.1) is cartesian.

(ii) The projection $\pi_{1}\left(X_{K}, \xi\right) \rightarrow G_{K}$ induces a natural isomorphism $\operatorname{Ker}(\mathrm{Sp}) \stackrel{\sim}{\rightarrow} I_{K}$ (i.e., maps $\operatorname{Ker}(\mathrm{Sp})$ isomorphically onto $\left.I_{K}\right)$.

Proof. The proof of (i) is similar to the proof of Lemma 3.3.2 in [Saïdi]. Assertion (ii) is clear in light of (i).

Let $s: G_{K} \rightarrow \pi_{1}\left(X_{K}, \xi\right)$ be a section of $\pi_{1}\left(X_{K}, \xi\right)$ so that it induces, by composing with the specialisation homomorphism $\mathrm{Sp}: \pi_{1}\left(X_{K}, \xi\right) \rightarrow \pi_{1}\left(X_{k}, \xi^{\prime}\right)$, a continuous homomorphism $s^{\prime} \stackrel{\text { def }}{=} \operatorname{Sp} \circ s: G_{K} \rightarrow \pi_{1}\left(X_{k}, \xi^{\prime}\right)$.

Lemma 2.1.2. The equality $\operatorname{Ker}\left(s^{\prime}\right)=I_{K}$ holds. In particular, $s^{\prime}$ factorizes through $G_{k}$ and induces a section $\tilde{s}: G_{k} \rightarrow \pi_{1}\left(X_{k}, \xi^{\prime}\right)$ of $\pi_{1}\left(X_{k}, \xi^{\prime}\right)$.

Proof. It suffices to show that the image $s^{\prime}\left(I_{K}\right)$ of the inertia subgroup in $\pi_{1}\left(X_{k}, \xi^{\prime}\right)$ is trivial. This image is contained in $\pi_{1}\left(X_{\bar{k}}, \bar{\xi}^{\prime}\right)$ by diagram (2.1). A standard (well-known) weight argument, using the fact that $k$ satisfies the condition (i) in Definition 0.2, shows that this image must be trivial (cf. [Hoshi-Mochizuki], Lemma $1.6)$.

Assume that the section $s$ is point-theoretic, i.e., $s=s_{x}: G_{K} \rightarrow \pi_{1}\left(X_{K}, \xi\right)$ is associated to a rational point $x \in X(K)$ (cf. $\S 0)$. Let $\bar{x} \in X(k)$ be the specialisation of the point $x$. Then one verifies easily that the section $\tilde{s}: G_{k} \rightarrow \pi_{1}\left(X_{k}, \xi^{\prime}\right)$ of $\pi_{1}\left(X_{k}, \xi^{\prime}\right)$ which is induced by $s$ (cf. Lemma 2.1.2) is point-theoretic and arises from the $k$-rational point $\bar{x}$, i.e., $[\tilde{s}]=[s \bar{x}]$ holds in $\overline{\operatorname{Sec}}_{\pi_{1}\left(X_{k}, \xi^{\prime}\right)}$. 
Lemma 2.1.3. We use the same notations as above. Let $x^{\prime} \in X(K)$ be a rational point which specialises in $\bar{x}$. Then $\left[s_{x}\right]=\left[s_{x^{\prime}}\right]$. In particular, the map $\varphi_{X}: X(K) \rightarrow$ $\overline{\mathrm{Sec}}_{\pi_{1}\left(X_{K}, \xi\right)}$ (cf. $\left.\S 0\right)$ is not injective.

Proof. Indeed, it follows immediately from Lemma 2.1.1(i) and Lemma 2.1.2 that a section $s: G_{K} \rightarrow \pi_{1}\left(X_{K}, \xi\right)$ of $\pi_{1}\left(X_{K}, \xi\right)$ is uniquely determined by the continuous homomorphism $s^{\prime} \stackrel{\text { def }}{=} \operatorname{Spos}: G_{K} \rightarrow G_{K} / I_{K} \rightarrow \pi_{1}\left(X_{k}, \xi^{\prime}\right)$ that it induces. In particular, all rational points $x^{\prime} \in X(K)$ which specialise in $\bar{x}$ (there are infinitely many such points $\left.x^{\prime}\right)$ give rise to the same conjugacy class of sections of $\pi_{1}\left(X_{K}, \xi\right)$, from which the second assertion follows.

Conversely we have the following.

Lemma 2.1.4. Assume that the section $\tilde{s}: G_{k} \rightarrow \pi_{1}\left(X_{k}, \xi^{\prime}\right)$ of $\pi_{1}\left(X_{k}, \xi^{\prime}\right)$ which is induced by $s$ (cf. Lemma 2.1.2) is point-theoretic, i.e., $\tilde{s}=s_{\bar{x}}$ for some $k$-rational point $\bar{x} \in X(k)$. Then the section $s$ is point-theoretic, i.e., $s=s_{x}$ for some (non unique) $x \in X(K)$ which specialises in the point $\bar{x}$.

Proof. Let $x \in X(K)$ be a rational point which specialises in $\bar{x}$ (such a point $x$ exists since $X$ is smooth). Then $[s]=\left[s_{x}\right]$ holds by the same argument used in the proof of Lemma 2.1.3.

2.2. The bad reduction case. In this section, and in addition to our assumptions, we will assume that $k$ satisfies the condition $(i v)(a)$ in Definition 0.2 unless we specify otherwise. We suppose that $X_{k}$ is singular. Recall that the irreducible components $\left\{X_{i}\right\}_{i \in I}$ of $X_{k}=\sum_{i \in I} X_{i}$ are smooth, geometrically connected, and the singular points $\left\{x_{j}\right\}_{j \in J}$ of $X_{k}$ are all $k$-rational.

Let $X_{i}$ be an irreducible component of $X_{k}$ and $D_{X_{i}} \subset \pi_{1}\left(X_{k}, \xi^{\prime}\right)^{\text {adm }}$ a decomposition group associated to $X_{i}$. Thus, $D_{X_{i}}$ is the decomposition group of an irreducible component of the special fibre of the universal admissible cover $\widetilde{X}$ of $X$ which lies above the component $X_{i}$, and $D_{X_{i}}$ is only defined up to conjugation (cf. [Mochizuki1], discussion before Proposition 4.1). Let $\bar{X}_{i}$ be the (unique, since $X_{i}$ is geometrically connected) irreducible component of $X_{\bar{k}}$ above $X_{i}$. Then we have the following commutative diagram.

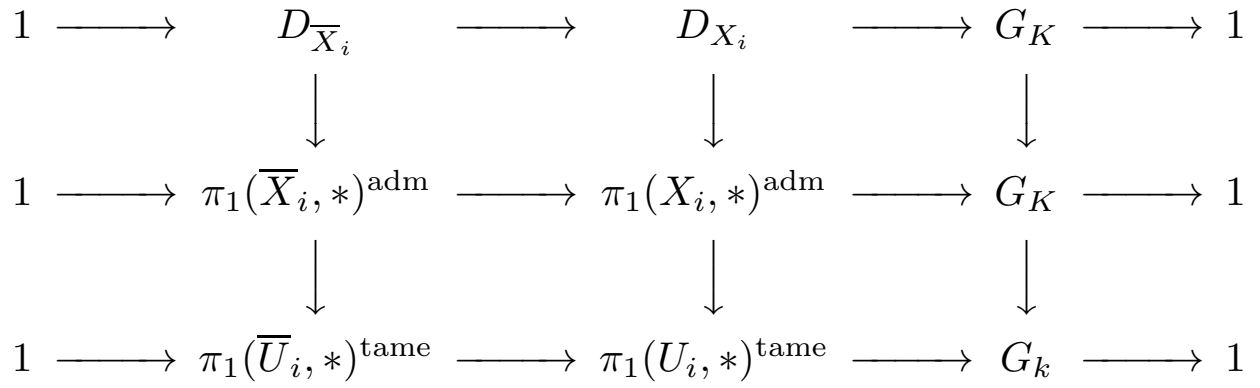

Here, $D_{\bar{X}_{i}}$ is defined so that the upper horizontal sequence is exact, $*$ denote suitable base points, and $\pi_{1}\left(X_{i}, *\right)^{\text {adm }}$ denotes the admissible fundamental group of $X_{i}$ which is marked by the cusps, i.e., the double points of $X_{k}$ lying on $X_{i}$ (cf. [Mochizuki1], Proposition 4.2 and the discussion before it which explains the log structure on $X_{i}$ and the choice of the base point $*$ involved in the definition of $\left.\pi_{1}\left(X_{i}, *\right)^{\mathrm{adm}}\right), \pi_{1}\left(\bar{X}_{i}, *\right)^{\text {adm }} \stackrel{\text { def }}{=} \operatorname{Ker}\left(\pi_{1}\left(X_{i}, *\right)^{\text {adm }} \rightarrow G_{K}\right), U_{i} \stackrel{\text { def }}{=} X_{i} \backslash\{$ cusps $\}$, and $\bar{U}_{i} \stackrel{\text { def }}{=} \bar{X}_{i} \backslash\{$ cusps $\}$, respectively. The superscript "tame" means tame fundamental 
group. The left and middle upper vertical maps are isomorphisms defined up to conjugation (cf. loc. cit.), and the lower right square is cartesian. Note that $\pi_{1}\left(\bar{U}_{i}, *\right)^{\mathrm{tame}}=\pi_{1}\left(\bar{U}_{i}, *\right)$, and $\pi_{1}\left(U_{i}, *\right)^{\mathrm{tame}}=\pi_{1}\left(U_{i}, *\right)$, since $\operatorname{char}(k)=0$.

Let $s: G_{K} \rightarrow \pi_{1}\left(X_{K}, \xi\right)$ be a section of $\pi_{1}\left(X_{K}, \xi\right)$, which induces a section $s^{\prime} \stackrel{\text { def }}{=} \mathrm{Spos}: G_{K} \rightarrow \pi_{1}\left(X_{k}, \xi^{\prime}\right)^{\mathrm{adm}}$ of the projection $\pi_{1}\left(X_{k}, \xi^{\prime}\right)^{\mathrm{adm}} \rightarrow G_{K}$ (cf. diagram (2.1)). Suppose that $X$ is regular and $s$ is point-theoretic, i.e., $s=s_{x}$ arises from a rational point $x \in X(K)$ (cf. $\S 0$ ). Then the $K$-rational point $x$ specialises in a rational point $\bar{x} \in X(k)$ which is a smooth point of $X_{k}$ and lies on a unique irreducible component $X_{i}$ of $X_{k}$ (cf. [Liu], Corollary 9.1.32). Moreover, it follows from the various definitions that $s\left(G_{K}\right) \subset D_{X_{i}} \subset \pi_{1}\left(X_{k}, \xi^{\prime}\right)^{\text {adm }}$ holds, where $D_{X_{i}}$ is a decomposition group associated to $X_{i}$ (cf. above discussion). In particular, the section $s=s_{x}$ induces a section $s_{i}: G_{K} \rightarrow \pi_{1}\left(X_{i}, *\right)^{\text {adm }}$ of the projection $\pi_{1}\left(X_{i}, *\right)^{\text {adm }} \rightarrow G_{K}$, and a continuous homomorphism $\tilde{s}_{i}: G_{K} \rightarrow \pi_{1}\left(U_{i}, *\right)^{\text {tame }}$ (cf. diagram (2.3)).

Lemma 2.2.1. The following hold.

(i) The section $s_{i}$ is unramified, i.e., $\tilde{s}_{i}\left(I_{K}\right)=\{1\}$. In particular, $\tilde{s}_{i}$ induces a section $\bar{s}_{i}: G_{k} \rightarrow \pi_{1}\left(U_{i}, *\right)^{\text {tame }}$ of $\pi_{1}\left(U_{i}, *\right)^{\text {tame }}$.

(ii) The section $\bar{s}_{i}: G_{k} \rightarrow \pi_{1}\left(U_{i}, *\right)^{\text {tame }}$ in (i) is point-theoretic and arises from the $k$-rational point $\bar{x} \in U_{i}(k)$. Moreover, the section $\bar{s}_{i}$ is non-cuspidal, i.e., $\bar{s}_{i}\left(G_{k}\right)$ is not contained in a decomposition group associated to a cusp.

Proof. Note that $U_{i}$ is hyperbolic since $X$ is stable. Assertion (i) follows from the assumptions (i) and (iv)(a). First, assumption (iv)(a) implies that the closed points of $X_{i}$ are uniquely determined by their corresponding (conjugacy classes of) decomposition groups in $\pi_{1}\left(U_{i}, *\right)^{\text {tame }}$, such a decomposition group is self-normalising in $\pi_{1}\left(U_{i}, *\right)^{\text {tame }}$, and no non-cuspidal decomposition group is contained in a cuspidal decomposition group (cf. the arguments used in the proof of Theorem $1.3 \mathrm{in}$ [Mochizuki2], and [Tamagawa], Proposition 2.8(i)). Second, if $\tilde{s}_{i}\left(I_{K}\right)$ is non trivial then one shows, using the condition (i), that $\tilde{s}_{i}\left(I_{K}\right) \subseteq \pi_{1}\left(\bar{U}_{i}, *\right)^{\text {tame }}$ would be a non-trivial (necessarily torsion-free) procyclic group contained in an inertia group $I_{y}$ at a cusp $y \in X_{i} \backslash U_{i}$ (cf. [Hoshi-Mochizuki], Lemma 1.6). Moreover, $\tilde{s}_{i}\left(G_{K}\right)$ (which normalises $\tilde{s}_{i}\left(I_{K}\right)$ ) is contained in a decomposition group associated to $y$ and the latter would contain a decomposition group associated to $\bar{x}$, which is a contradiction (cf. above discussion). Assertion (ii) follows easily.

Conversely, suppose that the section $s$ satisfies $s\left(G_{K}\right) \subset D_{X_{i}}$, i.e., the image of $s$ is contained in a decomposition group associated to an irreducible component $X_{i}$ of $X_{s}$. Thus, $s$ induces a section $s_{i}: G_{K} \rightarrow \pi_{1}\left(X_{i}, *\right)^{\text {adm }}$ of the projection $\pi_{1}\left(X_{i}, *\right)^{\text {adm }} \rightarrow G_{K}$ which induces a homomorphism $\tilde{s}_{i}: G_{K} \rightarrow \pi_{1}\left(U_{i}, *\right)^{\text {tame }}$ (cf. diagram (2.2)). Assume further that $s_{i}$ is unramified, i.e., $\tilde{s}_{i}\left(I_{K}\right)=\{1\}$. Then $\tilde{s}_{i}$ induces naturally a section $\bar{s}_{i}: G_{k} \rightarrow \pi_{1}\left(U_{i}, *\right)^{\text {tame }}$ of $\pi_{1}\left(U_{i}, *\right)^{\text {tame }}$ (cf. diagram $(2.2))$.

Lemma 2.2.2. With the same notations/assumptions as above suppose that the section $\bar{s}_{i}: G_{k} \rightarrow \pi_{1}\left(U_{i}, *\right)^{\text {tame }}$ is point-theoretic and arises from a rational point $\bar{x} \in U_{i}(k)$. Then the section $s$ is point-theoretic and arises from a (non unique) rational point $x \in X(K)$ which specialises in the point $\bar{x} \in U_{i}(k)$.

Proof. Similar to the proof of Lemma 2.1.4. 
2.3. Next, we provide examples of sections of arithmetic fundamental groups of hyperbolic curves over local fields of equal characteristic 0 which are not pointtheoretic. We use the notations and assumptions in 2.2. Let $X$ be a regular and stable $\mathcal{O}_{K}$-curve as above satisfying the conditions in 2.2. Let $x_{j} \in X(k)$ be a $k$-rational double point of $X_{k}$ and write $D_{x_{j}} \subset \pi_{1}\left(X_{k}, \xi^{\prime}\right)^{\text {adm }}$ for the decomposition group of $x_{j}$. Thus, $D_{x_{j}}$ is the decomposition group of a closed point of the special fibre of the universal admissible cover $\widetilde{X}$ of $X$ which lies above the double point $x_{j}$, and $D_{x_{j}}$ is only defined up to conjugation (cf. [Mochizuki1], $\S 5$ and $\S 6$ ). We have an exact sequence

$$
1 \rightarrow \Delta_{x_{j}} \rightarrow D_{x_{j}} \rightarrow G_{K} \rightarrow 1,
$$

where $\Delta_{x_{j}} \stackrel{\text { def }}{=} \operatorname{Ker}\left(D_{x_{j}} \rightarrow G_{K}\right)$. Moreover, there exists a natural isomorphism $\Delta_{x_{j}} \stackrel{\sim}{\rightarrow} \hat{\mathbb{Z}}(1)$ (cf. loc. cit.). The profinite group $D_{x_{j}}$ is isomorphic to the admissible fundamental group $\pi_{1}^{\text {adm }}(\mathcal{X})$ of $\mathcal{X} \stackrel{\text { def }}{=} \operatorname{Spec} \frac{\mathcal{O}_{K}[[S, T]]}{(S T-\pi)}$, where $\pi$ is a uniformiser of $\mathcal{O}_{K}$ (cf. loc. cit.). The above exact sequence splits. Indeed, the (admissible) covers $\mathcal{Y}_{N} \rightarrow \mathcal{X}$ defined generically by extracting an $\mathrm{N}$-th root of $S$ with $\mathcal{Y}_{N}$ normal, for all integers $N \geq 1$, define a splitting of this sequence. Such a splitting induces a section $s_{x_{j}}: G_{K} \rightarrow \pi_{1}\left(X_{K}, \xi\right) \stackrel{\sim}{\rightarrow} \pi_{1}\left(X_{k}, \xi^{\prime}\right)^{\mathrm{adm}}$ of $\pi_{1}\left(X_{K}, \xi\right)$. The section $s_{x_{j}}$ is not pointtheoretic. Indeed, if $s_{x_{j}}$ arises from a rational point $x \in X(K)$, then $x$ specialises in a smooth non-cuspidal point of an irreducible component $X_{i}$ (cf. the discussion before Lemma 2.2.1) which is necessarily adjacent to an irreducible component $X_{i^{\prime}}$ passing through $x_{j}$ (cf. [Hoshi-Mochizuki1], Corollary 1.15(iv)(c)(d)). Let $s_{i}: G_{k} \rightarrow \pi_{1}\left(U_{i}, *\right)^{\text {tame }}$ be the section of $\pi_{1}\left(U_{i}, *\right)^{\text {tame }}$ which is induced by $s_{x_{j}}$ (cf. Lemma 2.2.1.). This section (which is non-cuspidal) would then be cuspidal which contradicts Lemma 2.2.1(ii).

Proposition 2.3.1. Let $K$ be a complete discrete valuation field with residue field $k$ of characteristic 0. Assume that $k$ satisfies the conditions (i) and (iv)(a) (cf. Definition 0.2). Then there exists a flat, proper, smooth, geometrically connected hyperbolic curve $C$ over $K$, and a section $s: G_{K} \rightarrow \pi_{1}(C, *)$ of $\pi_{1}(C, *)$ which is not point-theoretic.

Proof. Write $\mathcal{O}_{K}$ for the valuation ring of $K$. Using formal patching techniques one can construct a proper, stable, and regular $\mathcal{O}_{K}$-curve $X$ satisfying the assumptions in 2.2. In particular, $C \stackrel{\text { def }}{=} X_{K}$ is smooth, hyperbolic, geometrically connected, and the double points of $X_{k}$ are $k$-rational (compare with [Saïdi1], Lemma 6.3). As in the above discussion (before Proposition 2.3.1), let $s_{x_{j}}: G_{K} \rightarrow \pi_{1}(C, *)$ be a section of $\pi_{1}(C, *)$ arising from a double point $x_{j}$ of the special fibre $X_{s}$ of $X$. Then $s_{x_{j}}$ is not point-theoretic as explained above.

$\S 3$. Sections of arithmetic fundamental groups of curves over function fields in characteristic 0 . We use the notations introduced in $\S 1$ and $\S 2$. In this section $k$ is a field of characteristic 0 which strongly satisfies the condition (i) in Definition 0.2. Let $C$ be a separated, smooth, and connected algebraic curve over $k$ with function field $K \stackrel{\text { def }}{=} k(C)$. Let $\mathcal{X} \rightarrow C$ be a flat, proper, and smooth relative curve with generic fibre $X \stackrel{\text { def }}{=} \mathcal{X} \times_{C}$ Spec $K$ which is geometrically connected and hyperbolic (i.e. $g(X) \geq 2$ ). Let $c \in C^{\text {cl }}$ be a closed point and $\mathcal{X}_{c} \stackrel{\text { def }}{=} \mathcal{X} \times_{C} \operatorname{Spec} k(c)$ the fibre of $\mathcal{X}$ at $c$. Let $\xi$ (resp. $\xi_{c}$ ) be a geometric point of $\mathcal{X}$ with value in its generic point (resp. with value in the generic point of $\mathcal{X}_{c}$ ). Then $\xi$ (resp. $\xi_{c}$ ) 
determines an algebraic closure $\bar{K}$ (resp. $\overline{k(c)}$ ) of $K$ (resp. of the residue field $k(c)$ of $C$ at $c$ ).

Lemma 3.1. For $c \in C^{\mathrm{cl}}$ there exists a commutative diagram

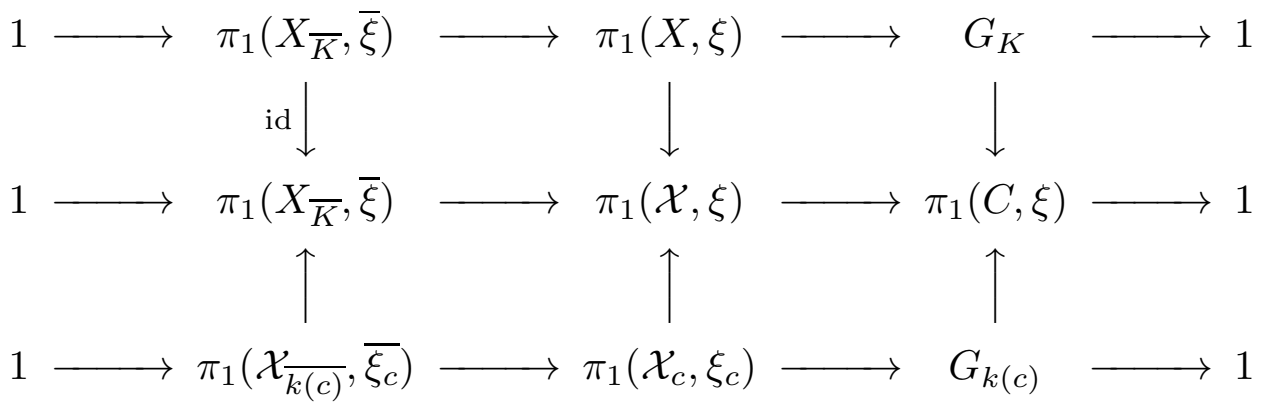

where $G_{K}=\operatorname{Gal}(\bar{K} / K), G_{k(c)}=\operatorname{Gal}(\overline{k(c)} / k(c)), \bar{\xi}$ (resp. $\overline{\xi_{c}}$ ) are geometric points induced by $\xi$ (resp. $\left.\xi_{c}\right)$, the middle and right upper vertical maps are natural continuous surjective homomorphisms, the middle lower vertical map is defined up to conjugation, the right lower vertical map is injective, and both squares on the right are cartesian.

Proof. Follows from the functoriality of fundamental groups and the homotopy exact sequence for $\pi_{1}$ (cf. [Grothendieck1], Éxposé XIII, §4).

Let $s: G_{K} \rightarrow \pi_{1}(X, \xi)$ be a section of $\pi_{1}(X, \xi)$.

Lemma 3.2. There exists a section $s_{C}: \pi_{1}(C, \xi) \rightarrow \pi_{1}(\mathcal{X}, \xi)$ of the projection $\pi_{1}(\mathcal{X}, \xi) \rightarrow \pi_{1}(C, \xi)$, which restricts for each closed point $c \in C^{\mathrm{cl}}$ to a section $s_{c}: G_{k(c)} \rightarrow \pi_{1}\left(\mathcal{X}_{c}, \xi_{c}\right)$ of $\pi_{1}\left(\mathcal{X}_{c}, \xi_{c}\right)$, and we have a commutative diagram

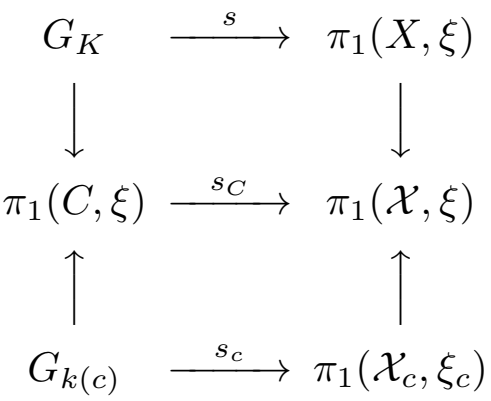

where the vertical maps are the ones in diagram (3.1).

Proof. Follows easily from Lemma 2.1.2 and Lemma 3.1.

From now on we assume that $X(K) \neq \emptyset$. Let $\mathcal{J} \stackrel{\text { def }}{=} \operatorname{Pic}_{\mathcal{X} / C}^{0} \rightarrow C$ be the (relative) jacobian of the (relative) curve $\mathcal{X} \rightarrow C, J \stackrel{\text { def }}{=} \mathcal{J}_{K}$ the jacobian variety of $X$, $\mathcal{J}_{c} \stackrel{\text { def }}{=} \mathcal{J}_{k(c)}$ the jacobian variety of $\mathcal{X}_{c}$, and $J_{c} \stackrel{\text { def }}{=} J_{K_{c}}$ the jacobian variety of $X_{K_{c}}$, where $c \in C^{\mathrm{cl}}$ and $K_{c}$ is the completion of $K$ at $c$. The commutative diagram (3.1) induces a commutative diagram of exact sequences of geometrically abelian 
fundamental groups (cf. §1)

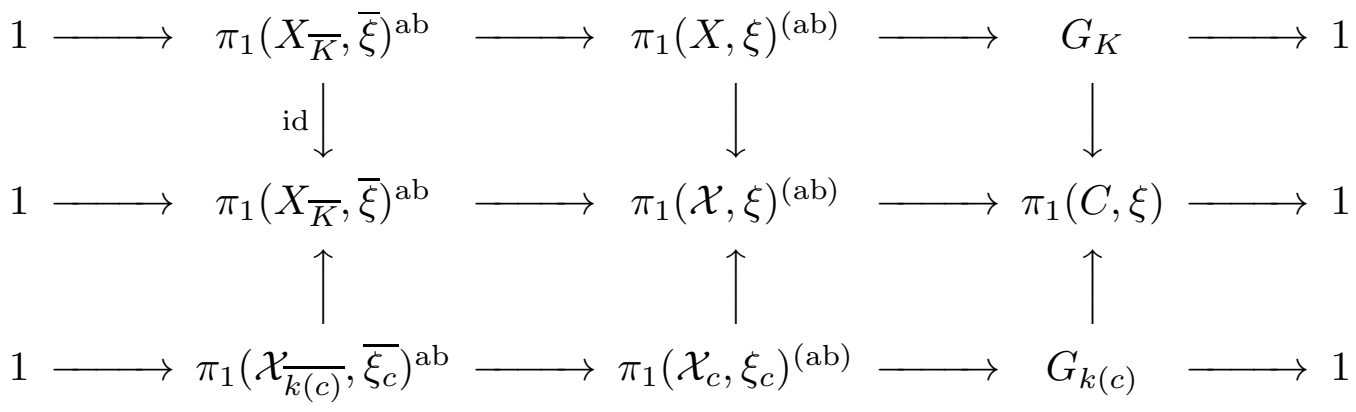

satisfying similar properties as in Lemma 3.1. Here $\pi_{1}(\mathcal{X}, \xi)^{(\mathrm{ab})}$ is defined as the push forward of the group extension $1 \rightarrow \pi_{1}\left(X_{\bar{K}}, \bar{\xi}\right) \rightarrow \pi_{1}(\mathcal{X}, \xi) \rightarrow \pi_{1}(C, \xi) \rightarrow 1$ by the characteristic quotient $\pi_{1}\left(X_{\bar{K}}, \bar{\xi}\right) \rightarrow \pi_{1}\left(X_{\bar{K}}, \bar{\xi}\right)^{\text {ab }}$. Fix a section $x \in \mathcal{X}(C)=$ $X(K)$ of $\mathcal{X} \rightarrow C$, and the embedding $\mathcal{X} \rightarrow \mathcal{J}$ mapping $x$ to the zero section. We identify $\pi_{1}\left(X_{\bar{K}}, \bar{\xi}\right)^{\text {ab }}\left(\right.$ resp. $\left.\pi_{1}\left(\mathcal{X}_{\overline{k(c)}}, \bar{\xi}_{c}\right)^{\text {ab }}\right)$ with the Tate module $T J$ (resp. $T \mathcal{J}_{c}$ ), and $T J_{c}$ with $T \mathcal{J}_{c}\left(K_{c}\right.$ is a complete discrete valuation ring of equal characteristic $0)$.

Lemma 3.3. Let $c \in C^{\mathrm{cl}}$. We have a commutative diagram of Kummer exact sequences (cf. $\S 1$ )

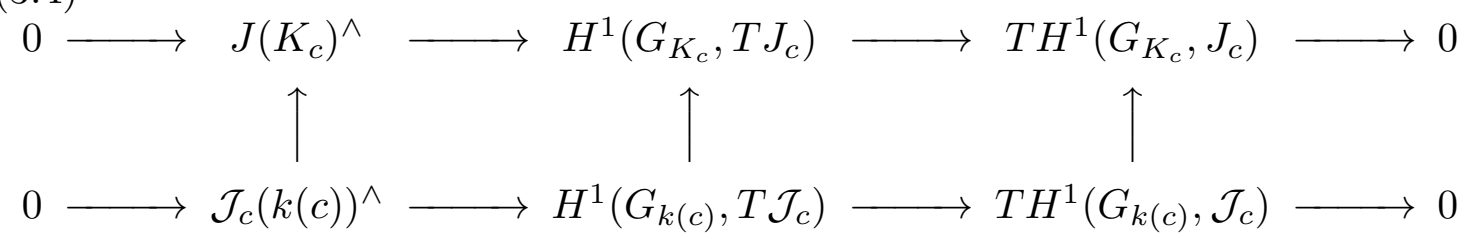

where the middle vertical map is the inflation map, the left vertical map is an isomorphism, the middle and right vertical maps are injective.

Proof. Follows from the fact that there exists an isomorphism $H^{1}\left(G_{k(c)}, T \mathcal{J}_{c}\right) \stackrel{\sim}{\rightarrow}$ $H^{1}\left(\operatorname{Gal}\left(K_{c}^{\mathrm{ur}} / K_{c}\right), T J_{c}\right)$ where $K_{c}^{\mathrm{ur}} / K_{c}$ denotes the maximal unramified subextension of $\overline{K_{c}} / K_{c}$, and the fact that the kernel of the specialisation map $J_{c}\left(K_{c}\right) \rightarrow$ $\mathcal{J}_{c}(k(c))$ is uniquely divisible (cf. [Lang-Tate], Proposition 8). (See also the commutative diagram in loc. cit. page 675.)

With the notations in Lemma 3.3, we will identify $H^{1}\left(G_{k(c)}, T \mathcal{J}_{c}\right)$ with its image in $H^{1}\left(G_{K_{c}}, T J_{c}\right)$. Next, we fix the base point of the torsor of splittings of the middle horizontal sequence in diagram (3.3) arising from the section $x \in \mathcal{X}(C)$, and the corresponding base points of the torsor of splittings of the upper and lower horizontal sequences in diagram (3.3). The sections $s: G_{K} \rightarrow \pi_{1}(X, \xi)$, $s_{C}: \pi_{1}(C, \xi) \rightarrow \pi_{1}(\mathcal{X}, \xi)$, and $s_{c}: G_{k(c)} \rightarrow \pi_{1}\left(\mathcal{X}_{c}, \xi_{c}\right)$ (cf. Lemma 3.2), give rise naturally to sections $s^{\mathrm{ab}}: G_{K} \rightarrow \pi_{1}(X, \xi)^{(\mathrm{ab})}, s_{C}^{\mathrm{ab}}: \pi_{1}(C, \xi) \rightarrow \pi_{1}(\mathcal{X}, \xi)^{(\mathrm{ab})}$, and $s_{c}^{\mathrm{ab}}: G_{k(c)} \rightarrow \pi_{1}\left(\mathcal{X}_{c}, \xi_{c}\right)^{(\mathrm{ab})}$, of the projections $\pi_{1}(X, \xi)^{(\mathrm{ab})} \rightarrow G_{K}, \pi_{1}(\mathcal{X}, \xi)^{(\mathrm{ab})} \rightarrow$ $\pi_{1}(C, \xi)$, and $\pi_{1}\left(\mathcal{X}_{c}, \xi_{c}\right)^{(\mathrm{ab})} \rightarrow G_{k(c)}$; respectively, which correspond to elements $s^{\mathrm{ab}} \in H^{1}\left(G_{K}, T J\right), s_{C}^{\mathrm{ab}} \in H^{1}\left(\pi_{1}(C, \xi), T J\right)$, and $s_{c}^{\mathrm{ab}} \in H^{1}\left(G_{k(c)}, T \mathcal{J}_{c}\right)$, for $c \in C^{\mathrm{cl}}$.

Lemma 3.4. Let $c \in C^{\mathrm{cl}}$. We have a commutative diagram of homomorphisms 


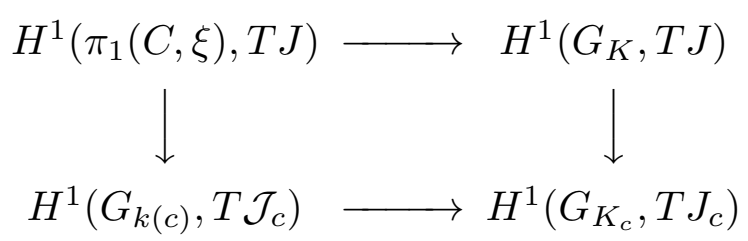

where the horizontal maps are injective inflation maps and the vertical maps are restriction homomorphisms, in which $s_{C}^{\mathrm{ab}}$ maps to $s^{\mathrm{ab}}$ (resp. $\left.s_{c}^{\mathrm{ab}}\right)$. In particular, the images of $s^{\mathrm{ab}}$ and $s_{c}^{\mathrm{ab}}$ in $H^{1}\left(G_{K_{c}}, T J_{c}\right)$ are equal. Moreover, assume that the section $s_{c}$ is point-theoretic, then $s_{c}^{\mathrm{ab}}$ is point-theoretic (cf. Definition 1.1(iii)). In particular, $s_{c}^{\mathrm{ab}}$ is pro-geometric both as an element of $H^{1}\left(G_{k(c)}, T \mathcal{J}_{c}\right)$ and $H^{1}\left(G_{K_{c}}, T J_{c}\right)$. (cf. Definition 1.1(i)).

Proof. The first assertion is easily verified. The second assertion follows from the various definitions (cf. Lemma 1.2), and Lemma 3.3.

Next, consider the following commutative diagram

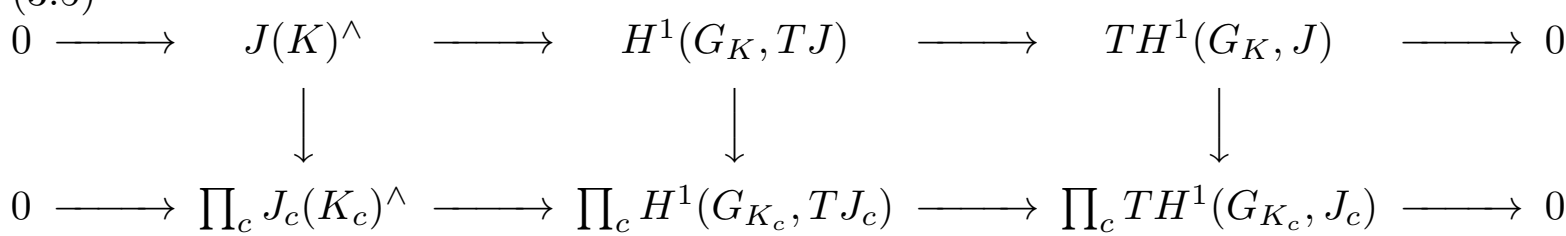

where the horizontal sequences are the Kummer exact sequences, the vertical maps are the diagonal mappings, and the product in the lower sequence is over all closed points $c \in C^{\mathrm{cl}}$. The image of $s^{\mathrm{ab}} \in H^{1}\left(G_{K}, T J\right)$ in $H^{1}\left(G_{K_{c}}, T J_{c}\right)$ via the above middle vertical map coincides with the element $s_{c}^{\mathrm{ab}} \in H^{1}\left(G_{k(c)}, T \mathcal{J}_{c}\right) \subset$ $H^{1}\left(G_{K_{c}}, T J_{c}\right), \forall c \in C^{\mathrm{cl}}$ (cf. Lemma 3.4). Recall (cf. $\S 0$ )

$$
\amalg(\mathcal{J})=\amalg(J, C) \stackrel{\text { def }}{=} \operatorname{Ker}\left(H^{1}\left(G_{K}, J\right) \rightarrow \prod_{c \in C^{\mathrm{cl}}} H^{1}\left(G_{K_{c}}, J_{c}\right)\right) .
$$

Note that the kernel of the map $T H^{1}\left(G_{K}, J\right) \rightarrow \prod_{c \in C} T H^{1}\left(G_{K_{c}}, J_{c}\right)$ in diagram (3.5) is the Tate module $T \amalg(\mathcal{J})$ of the Shafarevich-Tate group $\amalg(\mathcal{J})$. The following is immediate from the various definitions, and Lemma 3.4.

Lemma 3.5. Suppose that $s_{c}^{\mathrm{ab}} \in H^{1}\left(G_{K_{c}}, T J_{c}\right)$ is pro-geometric (cf. Definition 1.1(i)) $\forall c \in C^{\mathrm{cl}}$, and $T \amalg(\mathcal{J})=0$. Then $s^{\mathrm{ab}} \in J(K)^{\wedge}$ is pro-geometric.

$\S 4$. Proofs of Theorems A and B. Next, we prove Theorems A and B.

4.1. Proof of Theorem A. Recall the notations introduced in $\S 3$ that we will use throughout. Let $k, C, K=k(C), \mathcal{X} \rightarrow C$, and $X \rightarrow \operatorname{Spec} K$, be as in $\S 3$. We assume that $X(K) \neq \emptyset$, and $k$ strongly satisfies the conditions (i), (ii), (iv), (v), and (vi), in Definition 0.2. Let $\mathcal{J} \stackrel{\text { def }}{=} \operatorname{Pic}_{\mathcal{X} / C}^{0}$ be the relative jacobian of $\mathcal{X} \rightarrow C$, and assume that $T \amalg(\mathcal{J})=0$. We will show that the map (cf. $\S 0$ )

$$
\varphi_{X}: X(K) \rightarrow \overline{\operatorname{Sec}}_{\pi_{1}(X, \xi)}, \quad x \mapsto \varphi_{X}(x)=\left[s_{x}\right]
$$

is bijective.

First, we prove $\varphi_{X}$ is injective. Let $x_{1}, x_{2} \in X(K)$ such that $\left[s_{1} \stackrel{\text { def }}{=} s_{x_{1}}\right]=$ $\left[s_{2} \stackrel{\text { def }}{=} s_{x_{2}}\right]$ holds in $\overline{\operatorname{Sec}}_{\pi_{1}(X, \xi)}$. Thus, $\left[s_{1, c}\right]=\left[s_{2, c}\right]$ in $\overline{\operatorname{Sec}}_{\pi_{1}\left(\mathcal{X}_{c}, \xi_{c}\right)}, \forall c \in C^{\mathrm{cl}}$ (cf. 
Lemma 3.2), and $s_{i, c}=s_{x_{i, c}}$ is point-theoretic, where $x_{i, c} \in \mathcal{X}_{c}(k(c))$ is uniquely determined by $s_{i, c}$, for $i \in\{1,2\}$, as we assumed that $k$ strongly satisfies (ii). The $\operatorname{map} \varphi \mathcal{X}_{c}: \mathcal{X}_{c}(k(c)) \rightarrow \overline{\operatorname{Sec}}_{\pi_{1}\left(\mathcal{X}_{c}, \xi_{c}\right)}$ is bijective by assumption, hence $x_{1, c}=x_{2, c}$, $\forall c \in C^{\mathrm{cl}}$. Moreover, $x_{i, c}$ is the specialisation of $x_{i}$ in $\mathcal{X}_{c}$ (cf. discussion before Lemma 2.1.3). From this it follows that $x_{1}=x_{2}$ and $\varphi_{X}$ is injective. Indeed, the natural specialisation map $X(K) \rightarrow \prod_{c \in C^{\mathrm{cl}}} \mathcal{X}_{c}(k(c))$ is injective.

Next, we prove that $\varphi_{X}$ is surjective. Let

$$
s: G_{K} \rightarrow \pi_{1}(X, \xi)
$$

be a section of $\pi_{1}(X, \xi)$. We will show that $s$ is point-theoretic under the above assumptions. First, we have a diagram (3.2) and it follows from the condition (ii) that the section $s_{c}$ (cf. loc. cit.) is point-theoretic and arises from a unique rational point $x_{c} \in \mathcal{X}(k(c)), \forall c \in C^{\text {cl }}$. Moreover, $s_{c}^{\mathrm{ab}} \in J\left(K_{c}\right)^{\wedge} \subset H^{1}\left(G_{K_{c}}, T J_{c}\right)$ is pro-geometric in the sense of Definition 1.1(i), $\forall c \in C^{\text {cl }}$ (cf. Lemma 3.4). Then it follows from the assumption $T \amalg(\mathcal{J})=0$ that $s^{\text {ab }} \in J(K)^{\wedge} \subset H^{1}\left(G_{K}, T J\right)$ is pro-geometric (cf. Lemma 3.5).

Lemma 4.1.1. The natural homomorphism $J(K) \rightarrow J(K)^{\wedge}$ is injective and $s^{\mathrm{ab}} \in$ $J(K) \subseteq J(K)^{\wedge}$ is geometric.

Proof. There exist closed points $c_{1}, c_{2} \in C^{\mathrm{cl}}$ such that the natural specialisation homomorphism $J(K) \rightarrow \mathcal{J}_{c_{1}}\left(k\left(c_{1}\right)\right) \times \mathcal{J}_{c_{2}}\left(k\left(c_{2}\right)\right)$ is injective (cf. [Poonen-Voloch], Proposition 2.4). We have a commutative diagram of exact sequences

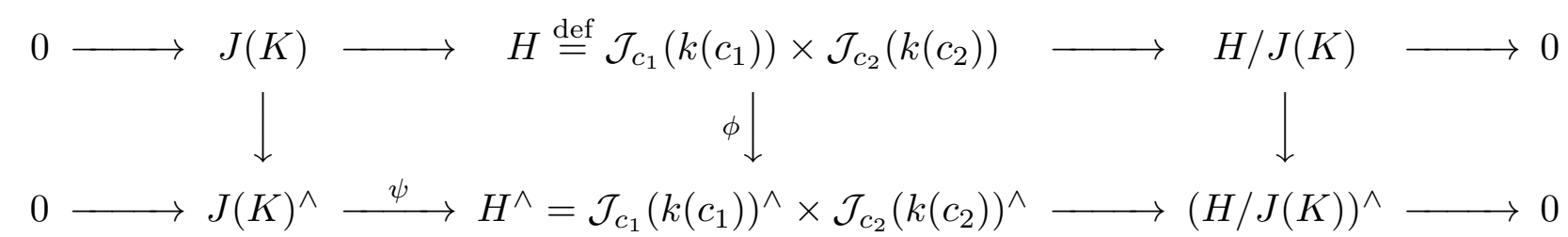

where the right and middle vertical maps are injective by the assumption (iv)(a), and the maps $\psi$ and $\phi$ are the natural ones. (The exactness of the lower sequence in the above diagram follows easily from the assumption (iv)(b).) In particular, the left vertical map is injective, and the equality $J(K)=\phi(H) \cap \psi\left(J(K)^{\wedge}\right)$ holds inside $H^{\wedge}$. The image of $s^{\mathrm{ab}} \in J(K)^{\wedge}$ in $H^{\wedge}$ via the map $\psi$ is the element $\left(s_{c_{1}}^{\mathrm{ab}}, s_{c_{2}}^{\mathrm{ab}}\right) \in$ $H^{\wedge} \subset H^{1}\left(G_{k\left(c_{1}\right)}, T \mathcal{J}_{c_{1}}\right) \times H^{1}\left(G_{k\left(c_{2}\right)}, T \mathcal{J}_{c_{2}}\right)$ associated to the sections $s_{c_{i}}: G_{k\left(c_{i}\right)} \rightarrow$ $\pi_{1}\left(\mathcal{X}_{c_{i}}, \xi_{c_{i}}\right)$ (induced by the section $s$ ), for $i \in\{1,2\}$, which are point-theoretic by the assumption (ii). In particular, $s_{c_{i}}^{\text {ab }} \in \mathcal{J}_{c_{i}}\left(k\left(c_{i}\right)\right)$ is geometric (cf. Lemma 1.2), and $s^{\mathrm{ab}} \in J(K)$ is geometric by the above discussion.

We fix an embedding $\mathcal{X} \stackrel{\iota}{\rightarrow} \mathcal{J}$ mapping an element of $\mathcal{X}(C)=X(K)$ (which is non-empty by our assumptions) to the zero section.

Lemma 4.1.2. The element $s^{\mathrm{ab}} \in \iota(X(K)) \subset J(K)$ is point-theoretic.

Proof. For each closed point $c \in C^{\text {cl }}$ the element $s_{c}^{\text {ab }} \in \mathcal{J}(k(c))^{\wedge} \subset H^{1}\left(G_{k(c)}, T \mathcal{J}_{c}\right)$ corresponding to the section $s_{c}: G_{k(c)} \rightarrow \pi_{1}\left(\mathcal{X}_{c}, \xi_{c}\right)$ lies in the subset $\iota(\mathcal{X}(k(c))) \subset$ $\mathcal{J}(k(c)) \subset \mathcal{J}(k(c))^{\wedge}$, since the section $s_{c}$ is point-theoretic by (ii). We view $s^{\text {ab }} \in$ $J(K)$ as a rational section of $\mathcal{J} \rightarrow C$; in fact $s^{\text {ab }}: C \rightarrow \mathcal{J}$ is a morphism since $C$ is a smooth curve. For each closed point $c \in C^{\mathrm{cl}}$ the image $s^{\mathrm{ab}}(c)$ is a closed point of 
$\mathcal{X}_{c} \subset \mathcal{J}_{c}$, where we view $\mathcal{X}_{c}$ as a closed subscheme of $\mathcal{J}_{c}$ via the closed immersion $\mathcal{X}_{c} \stackrel{\iota_{c}}{\longrightarrow} \mathcal{J}_{c}$ induced by $\iota$. From this it follows that the morphism $s^{\text {ab }}: C \rightarrow \mathcal{J}$ factorizes as $s^{\mathrm{ab}}: C \rightarrow \mathcal{X} \stackrel{\iota}{\rightarrow} \mathcal{J}$ and $s^{\mathrm{ab}}$ belongs to the subset $\iota(X(K)) \subseteq J(K)$.

Let $\tilde{x} \in X(K)$ such that $\iota(\tilde{x})=s^{\text {ab }}$ (cf. Lemma 4.1.2). For $c \in C^{\mathrm{cl}}$ let $\tilde{x}_{c}$ be the specialisation of $\tilde{x}$ in $\mathcal{X}_{c}$.

Lemma 4.1.3. The equality $\tilde{x}_{c}=x_{c}$ holds in $\mathcal{X}(k(c)), \forall c \in C^{\mathrm{cl}}$.

Proof. First, the equality $s_{c}^{\mathrm{ab}}=s_{\tilde{x}_{c}}^{\mathrm{ab}}=s_{x_{c}}^{\mathrm{ab}}$ holds in $H^{1}\left(G_{k(c)}, T \mathcal{J}_{c}\right)$ (cf. Lemma 3.4). The equality $\tilde{x}_{c}=x_{c}$ follows then from the injectivity of the maps $\iota\left(\mathcal{X}_{c}\right) \hookrightarrow$ $\mathcal{J}_{c}(k(c)) \hookrightarrow \mathcal{J}_{c}(k(c))^{\wedge} \rightarrow H^{1}\left(G_{k(c)}, T \mathcal{J}_{c}\right)$, for $c \in C^{\text {cl }}$ (see the assumption (iv)(a)).

Next, and in order to show that the section $s$ is point-theoretic it suffices to show, by a well-know limit argument in anabelian geometry (cf. [Tamagawa], Proposition 2.8 (iv)), using the assumption (v) (cf. Definition 0.2), the following. Let $H \subseteq$ $\pi_{1}(X, \xi)$ be an open subgroup such that $s\left(G_{K}\right) \subset H$, corresponding to an étale cover $Y \rightarrow X$, then $Y(K) \neq \emptyset$ holds. There is a natural identification $H=\pi_{1}(Y, \xi)$. Moreover, the cover $Y \rightarrow X$ extends to an étale cover $\mathcal{Y} \rightarrow \mathcal{X}$ and $\mathcal{Y}$ is a smooth model of $Y$ over $C$ (cf. Lemma 3.1 and Lemma 3.2). For a closed point $c \in C^{\mathrm{cl}}$ write $\mathcal{Y}_{c} \stackrel{\text { def }}{=} \mathcal{Y}_{k(c)}$. We will show that $Y(K) \neq \emptyset$.

Let $s^{\prime}: G_{K} \rightarrow \pi_{1}(Y, \xi)$ be the section of $\pi_{1}(Y, \xi)=H$ induced by $s$, which extends to a section $s^{\prime}: \pi_{1}(C, \xi) \rightarrow \pi_{1}(\mathcal{Y}, \xi)$ of the projection $\pi_{1}(\mathcal{Y}, \xi) \rightarrow \pi_{1}(C, \xi)$, and further induces a section $s_{c}^{\prime}: G_{k(c)} \rightarrow \pi_{1}\left(\mathcal{Y}_{c}, \xi_{c}\right)$ of $\pi_{1}\left(\mathcal{Y}_{c}, \xi_{c}\right), \forall c \in C^{\text {cl }}$ (cf. loc. cit.). Note that $s_{c}^{\prime}$ is induced by the section $s_{c}$. The section $s_{c}^{\prime}$ is point-theoretic and arises from a unique rational point $y_{c} \in \mathcal{Y}_{c}(k(c))$. Moreover, $x_{c}$ is the image of $y_{c}$ in $\mathcal{X}_{c}$ via the morphism $\mathcal{Y}_{c} \rightarrow \mathcal{X}_{c}$ (cf. condition (ii), the fact that $s_{c}$ is pointtheoretic and arises from $x_{c}$, and $s_{c}^{\prime}$ is induced by $s_{c}$ ). View $\tilde{x} \in X(K)=\mathcal{X}(C)$ as a section $\tilde{x}: C \rightarrow \mathcal{X}$, and let $\mathcal{Y}_{\tilde{x}}$ be the scheme-theoretic inverse image of $\tilde{x}(C)$ in $\mathcal{Y}$ via the above étale map $\mathcal{Y} \rightarrow \mathcal{X}$. Thus, $\mathcal{Y}_{\tilde{x}} \rightarrow \tilde{x}(C)$ is a finite étale map. We have $y_{c} \in \mathcal{Y}_{\tilde{x}}(k(c)), \forall c \in C^{c l}$, as follows from the various definitions. Then $\mathcal{Y}_{\tilde{x}}(K) \neq \emptyset$ by the assumption (vi), and a fortiori $\mathcal{Y}_{\tilde{x}}(K) \subseteq \mathcal{Y}(K)=Y(K) \neq \emptyset$.

Thus, we proved that $[s]=\left[s_{x}\right]$ holds in $\overline{\operatorname{Sec}}_{\pi_{1}(X, \xi)}$ for a (unique) $x \in X(K)$. The following follows from Lemma 4.1.3 (cf. above proof that $\varphi_{X}$ is injective).

Lemma 4.1.4. The equality $x=\tilde{x}$ holds.

This finishes the proof of Theorem A.

Finally, we show that Hilbertian fields satisfy the condition (vi).

Lemma 4.1.5. Let $k$ be a Hilbertian field. Then $k$ strongly satisfies the condition (vi).

Proof. Let $k^{\prime} / k$ be a finite extension and $C$ a separated, smooth, and connected curve over $k^{\prime}$ with function field $K \stackrel{\text { def }}{=} k^{\prime}(C)$. Let $\widetilde{C} \rightarrow C$ be a finite étale cover with $\widetilde{C}_{c}\left(k^{\prime}(c)\right) \neq \emptyset, \forall c \in C^{\text {cl }}$ with residue field $k^{\prime}(c)$. Note that $k^{\prime}$ is Hilbertian (cf. [Serre], 9.5). We show $\widetilde{C}(K) \neq \emptyset$. Assume that $\widetilde{C}(K)=\emptyset$. Then for each connected component $\widetilde{C}_{\alpha}$ of $\widetilde{C}$ the degree of the morphism $\widetilde{C}_{\alpha} \rightarrow C$ is $\geq 2$. Hilbert's irreducibility Theorem (cf. [Serre], 9.2) implies that there exist points $c \in C^{\mathrm{cl}}$ such that the fibre of $c$ in each connected component of $\widetilde{C}$ is irreducible. This contradicts the assumption that $\widetilde{C}_{c}(k(c)) \neq \emptyset, \forall c \in C^{\mathrm{cl}}$. Thus, $\widetilde{C}(K) \neq \emptyset$ holds. 
In the course of proving Theorem A we proved the following "adelic MordellLang" statement.

Proposition 4.1.6. With the same notations as above, assume that $k$ only satisfies the condition (iv) in Definition 0.2 (where we take $k^{\prime}=k$ ), and $\mathcal{X}(C) \neq$ $\emptyset$. Then the map $J(K)^{\wedge} \rightarrow \prod_{c \in C^{\mathrm{cl}}} J_{c}\left(K_{c}\right)^{\wedge} \stackrel{\sim}{\rightarrow} \prod_{c \in C^{\mathrm{cl}}} \mathcal{J}_{c}(k(c))^{\wedge}$ (cf. diagram (3.4) and Lemma 3.3) is injective. Further, inside $\prod_{c \in C^{\mathrm{cl}}} \mathcal{J}_{c}(k(c))^{\wedge}$ the equality $J(K)^{\wedge} \cap\left(\prod_{c \in C^{\mathrm{cl}} \mathrm{l}} \iota\left(\mathcal{X}_{c}(k(c))\right)\right)=\iota(X(K))$ holds.

Proof. See the statements and proofs of lemma 4.1.1 and Lemma 4.1.2.

4.2. Proof of Theorem B. We briefly explain how Theorem B can be derived from Theorem A. Let $k$ be a field with char $(k)=0$, and $K=k(C)$ the function field of a separated, smooth, and connected curve $C$ over $k$. Assume that $k$ satisfies the condition $(\star)$ (cf. Definition 0.2 ). Let $L / K$ be a finite extension. Then we prove the SC holds over $L$. We can, without loss of generality, assume that $L=K$. Let $X$ be a proper, smooth, and hyperbolic curve over $K$, we need to prove that the $\mathbf{S C}$ holds for $X$, i.e., that the map (cf. $\S 0) \varphi_{X}: X(K) \rightarrow \overline{\operatorname{Sec}}_{\pi_{1}(X, \xi)}$ is bijective. The injectivity of the map $\varphi_{X}$ follows as in the proof of Theorem A, where one only uses the fact that $k$ strongly satisfies the condition (ii) (cf. loc. cit.)

Next, we prove that $\varphi_{X}$ is surjective. Let $s: G_{K} \rightarrow \pi_{1}(X, \xi)$ be a section of $\pi_{1}(X, \xi)$. We need to show that $s$ is point-theoretic under the above assumptions. For this purpose we can, in the course of the proof, replace $K$ by a finite extension $L^{\prime} / K$. Indeed, let $L^{\prime} / K$ be a finite Galois extension, $s^{\prime}: G_{L^{\prime}} \stackrel{\text { def }}{=} \operatorname{Gal}\left(\bar{K} / L^{\prime}\right) \rightarrow$ $\pi_{1}\left(X_{L^{\prime}}, \xi\right)$ the section of $\pi_{1}\left(X_{L^{\prime}}, \xi\right)$ which is induced by $s$, and assume that $s^{\prime}=s_{x^{\prime}}$ is point-theoretic where $x^{\prime} \in X\left(L^{\prime}\right)$. Then $s^{\prime}\left(G_{L^{\prime}}\right)$ is self-normalising in $\pi_{1}\left(X_{L^{\prime}}, \xi\right)$, and $s\left(G_{K}\right)$ is contained in the normaliser of $s^{\prime}\left(G_{L^{\prime}}\right)$ in $\pi_{1}(X, \xi)$ which coincides with a decomposition group associated to the image $x$ of $x^{\prime}$ in $X$ (this follows from condition $(\star)(i v)(a)$, cf. proof of Lemma 2.2.1 and the references therein). The point $x$ is then necessarily $K$-rational. Now consider a finite (Galois) extension $L^{\prime} / K$ such that $X\left(L^{\prime}\right) \neq \emptyset$, then the corresponding section $s^{\prime}: G_{L^{\prime}} \stackrel{\text { def }}{=} \operatorname{Gal}\left(\bar{K} / L^{\prime}\right) \rightarrow$ $\pi_{1}\left(X_{L^{\prime}}, \xi\right)$ of $\pi_{1}\left(X_{L^{\prime}}, \xi\right)$ which is induced by $s$ is point-theoretic by Theorem $\mathrm{A}$ and our assumption that $k$ satisfies the condition $(\star)$. From this it follows that $s$ is point theoretic (cf. above discussion).

$\S 5$. Sections of arithmetic fundamental groups of curves over finitely generated fields in characteristic 0. In this section we combine the techniques/results in $\S 3$ and $\S 4$ with the results in [Saïdi-Tamagawa] to prove variants of Theorems A and B, as well as Theorem C. We use the notations introduced in previous sections. All fields in this section are finitely generated over $\mathbb{Q}$. In order to deduce the validity of the $\mathbf{S C}$ over finitely generated fields from its validity over number fields (which for the time being is still not known), one is reduced (by an induction argument) to prove the following.

Conjecture D. Assume that the SC holds over all finitely generated fields of transcendence degree $i \geq 0$. then the $\mathbf{S C}$ holds over all finitely generated fields of transcendence degree $i+1$.

Next, we recall the discrete Selmer conjecture formulated in [Saïdi-Tamagawa]. Let $k$ be a finitely generated field over $\mathbb{Q}, C$ a separated, smooth, and geometrically connected curve over $k, K \stackrel{\text { def }}{=} k(C)$ the function field of $C$, and

$$
\mathcal{A} \rightarrow C
$$


an abelian scheme over $C$. Write $A \stackrel{\text { def }}{=} \mathcal{A} \times{ }_{C} \operatorname{Spec} K$ for the generic fibre of $\mathcal{A}$. For a closed point $c \in C^{\text {cl }}$, let $k(c)$ be the residue field at $c$, and $\mathcal{A}_{c} \stackrel{\text { def }}{=} \mathcal{A}_{k(c)}=$ $\mathcal{A} \times{ }_{C} \operatorname{Spec} k(c)$. Then we have a commutative diagram of exact sequences

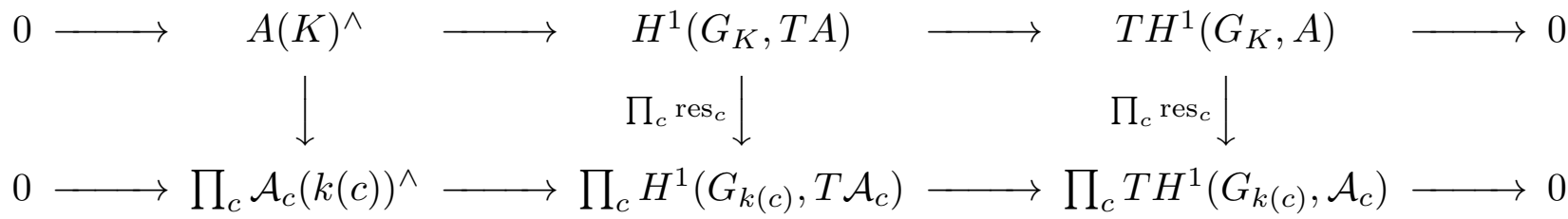

where the horizontal sequences are the Kummer exact sequences, the vertical maps are natural restriction homomorphisms, and the product is taken over all closed points $c \in C^{\mathrm{cl}}$ (cf. loc. cit. diagram (0.1)). Define the profinite Selmer group

$$
\operatorname{Sel}(\mathcal{A}) \stackrel{\text { def }}{=} \operatorname{Sel}(A, C) \stackrel{\text { def }}{=} \operatorname{Ker}\left(H^{1}\left(G_{K}, T A\right) \rightarrow \prod_{c} T H^{1}\left(G_{k(c)}, \mathcal{A}_{c}\right)\right)
$$

and the Shafarevich-Tate group

$$
\amalg(\mathcal{A}) \stackrel{\text { def }}{=} \amalg(A, C) \stackrel{\text { def }}{=} \operatorname{Ker}\left(H^{1}\left(G_{K}, A\right) \rightarrow \prod_{c} H^{1}\left(G_{k(c)}, \mathcal{A}_{c}\right)\right),
$$

where the product is taken over all closed points $c \in C^{\mathrm{cl}}$. For each $c \in C^{\mathrm{cl}}$ the group $\mathcal{A}(k(c))$ of $k(c)$-rational points of $\mathcal{A}$ is finitely generated as $k(c)$ is finitely generated over $\mathbb{Q}$ (Mordell-Weil Theorem), hence injects into its profinite completion $\mathcal{A}(k(c))^{\wedge}$. We identify $\mathcal{A}(k(c))$ with its image in $\mathcal{A}(k(c))^{\wedge}$. Define the discrete Selmer group by

$$
\operatorname{Sel}(\mathcal{A}) \stackrel{\text { def }}{=} \operatorname{Sel}(A, C) \stackrel{\text { def }}{=} \operatorname{Sel}(\mathcal{A}) \bigcap \prod_{c} \mathcal{A}(k(c)) \subset \prod_{c} H^{1}\left(G_{k(c)}, T \mathcal{A}_{c}\right),
$$

where the product is taken over all closed points $c \in C^{\mathrm{cl}}$. Note that $A(K) \subseteq \mathfrak{S e l}(\mathcal{A})$. In [Saïdi-Tamagawa] we conjectured the following (cf. loc. cit. Conjecture E).

Conjecture E. The equality $\operatorname{Sel}(\mathcal{A})=A(K)$ holds.

Moreover, we proved the following facts.

Facts. (i) $\operatorname{Sel}(\mathcal{A})$ is a finitely generated abelian group (cf. loc. cit. Proposition $2.5)$.

(ii) Assume that there exists a prime integer $\ell$ such that the $\ell$-primary part $\amalg(\mathcal{A})\left[\ell^{\infty}\right] \stackrel{\text { def }}{=} \bigcup_{n \geq 1} \amalg(\mathcal{A})\left[l^{n}\right]$ of $\amalg(\mathcal{A}) \stackrel{\text { def }}{=} \amalg(A, C)$ is finite. Then $\operatorname{Sel}(\mathcal{A})=$ $A(K)$, i.e., Conjecture E holds in this case (cf. loc. cit. Proposition 3.7).

(iii) Assume that $A$ is isotrivial, i.e., $A_{\bar{K}}$ is defined over $\bar{k}$, then $\amalg(\mathcal{A})$ is finite (cf. loc. cit. Theorem 4.2). In particular, $\mathfrak{S e l}(\mathcal{A})=A(K)$ holds, i.e., Conjecture $\mathrm{E}$ holds in this case (cf. above fact (ii)).

One of our main results in this paper is a (conditional) proof of Conjecture D. More precisely, we prove the following (cf. discussion after Corollary 5.3). 
Theorem 5.1. Assume that Conjecture $\mathbf{E}$ holds. Then Conjecture $\mathbf{D}$ holds true.

In other words, Theorem 5.1 asserts that the validity of the above discrete Selmer conjecture implies that one can reduce the section conjecture $\mathbf{S C}$ over all finitely generated fields to the case of number fields. The following Corollaries follow immediately from Theorem 5.1, and the above cited Facts (ii), and (iii); respectively. (For the proof of Corollary 5.3, use an induction argument.)

Corollary 5.2. Assume that the following holds. Given a finitely generated field $k$ over $\mathbb{Q}$, a separated, smooth, and connected curve $C$ over $k$ with generic point $\eta$, and an abelian scheme $\mathcal{A} \rightarrow C$ with generic fibre $A \stackrel{\text { def }}{=} \mathcal{A} \times_{C} \eta$, then there exists a prime integer $\ell$ such that the $\ell$-primary part $\amalg(\mathcal{A})\left[\ell^{\infty}\right] \stackrel{\text { def }}{=} \bigcup_{n \geq 1} \amalg(\mathcal{A})\left[l^{n}\right]$ of $\amalg(\mathcal{A}) \stackrel{\text { def }}{=} \amalg(A, C)$ is finite. Then Conjecture $\mathbf{D}$ holds true.

Corollary 5.3. Let $K$ be a finitely generated field with algebraic closure $\bar{K}$ and $\overline{\mathbb{Q}}$ the algebraic closure of $\mathbb{Q}$ in $\bar{K}$. Assume that the SC holds over all number fields. Then the $\mathbf{S C}$ holds for every projective smooth and geometrically connected hyperbolic curve $X$ over $K$ such that $X \times_{\operatorname{Spec} K} \operatorname{Spec} \bar{K}$ is defined over $\overline{\mathbb{Q}}$.

The rest of this section is devoted to the proof of Theorem 5.1. To this end it suffices to prove the following (compare with Theorem A). (Note that the assumption $X(K) \neq \varnothing$ in Theorem 5.4 is not restrictive in order to deduce Theorem 5.1 from Theorem 5.4 (cf. arguments in the proof of Theorem B).)

Theorem 5.4. Assume that the SC holds over all finitely generated fields of transcendence degree $i \geq 0$. Let $k$ be a field with $\operatorname{tr} \operatorname{deg}_{\mathbb{Q}} k=i$, and $K=k(C)$ the function field of a separated, smooth, and connected curve $C$ over $k$. Let $\mathcal{X} \rightarrow C$ be a flat, proper, and smooth relative curve with generic fibre $X \stackrel{\text { def }}{=} \mathcal{X} \times_{C} \operatorname{Spec} K$ which is hyperbolic and geometrically connected, $J \stackrel{\text { def }}{=} \operatorname{Pic}_{X / K}^{0}$, and $\mathcal{J} \stackrel{\text { def }}{=} \operatorname{Pic}_{\mathcal{X} / C}^{0}$. Assume that $X(K) \neq \emptyset$, and either there exists a prime integer $\ell$ such that the $\ell$-primary part $\amalg(\mathcal{J})\left[\ell^{\infty}\right]$ of $\amalg(\mathcal{J}) \stackrel{\text { def }}{=} \amalg(J, C)$ is finite or $\operatorname{Sel}(\mathcal{J}) \stackrel{\text { def }}{=} \mathfrak{S e l}(J, C)=J(K)$ holds. Then the SC holds for $X$ (over $K)$.

Proof. The proof of the injectivity of the map $\varphi_{X}: X(K) \rightarrow \overline{\operatorname{Sec}}_{\pi_{1}(X, \xi)}$ is similar to the proof of injectivity in the statement of Theorem A (cf. discussion before Lemma 4.1.1). In what follows we prove $\varphi$ is surjective. Let $s: G_{K} \rightarrow \pi_{1}(X, \xi)$ be a section of $\pi_{1}(X, \xi)$. We will show that $s$ is point-theoretic under the above assumptions. We use the notations in $\S 3$. Recall that $s$ induces a section $s_{c}: G_{k(c)} \rightarrow \pi_{1}\left(\mathcal{X}_{c}, \xi_{c}\right)$ of $\pi_{1}\left(\mathcal{X}_{c}, \xi_{c}\right), \forall c \in C^{\mathrm{cl}}$ (cf. Lemma 3.2). By our assumption that the $\mathbf{S C}$ holds over all finitely generated fields of transcendence degree $i$ we deduce that the section $s_{c}$ is point-theoretic and arises from a unique rational point $x_{c} \in \mathcal{X}(k(c)), \forall c \in C^{\mathrm{cl}}$. The element $s_{c}^{\mathrm{ab}} \in H^{1}\left(G_{k(c)}, T \mathcal{J}_{c}\right)$ is then point-theoretic, hence is geometric (cf. Lemma 1.2). Thus, $s_{c}^{\mathrm{ab}} \in \mathcal{J}(k(c)) \subseteq \mathcal{J}(k(c))^{\wedge} \subseteq H^{1}\left(G_{k(c)}, T \mathcal{J}_{c}\right), \forall c \in C^{\mathrm{cl}}$. In particular, $s^{\mathrm{ab}} \in \mathfrak{S e l}(\mathcal{J}) \stackrel{\text { def }}{=} \mathfrak{S e l}(J, C)$ is in the discrete Selmer group. By our second assumption, that either there exists a prime integer $\ell$ such that the $\ell$-primary part $\amalg(\mathcal{J})\left[\ell^{\infty}\right]$ of $\amalg(\mathcal{J}) \stackrel{\text { def }}{=} \amalg(J, C)$ is finite or $\operatorname{Sel}(\mathcal{J}) \stackrel{\text { def }}{=} \operatorname{Sel}(J, C)=J(K)$ holds (note that the former condition implies the latter one by Fact (ii) above), we deduce that $s^{\mathrm{ab}} \in J(K)$ is geometric. Moreover, a similar proof to that of Lemma 4.1.2 shows that $s^{\text {ab }}$ lies in the subset $X(K)$ of $J(K)$ (cf. loc. cit.). The rest of the proof is similar to that of Theorem A (cf. Lemma 4.1.3 and Lemma 4.1.4). 
This finishes the proof of Theorem 5.4, and Theorem 5.1.

Remark 5.5. The condition in Theorem 5.4 involving $\amalg(\mathcal{J})\left[\ell^{\infty}\right]$ is weaker than the condition in Theorem A and Corollary A involving $\amalg(\mathcal{J})$.

\section{References.}

[Cadoret-Tamagawa] Torsion of abelian schemes and rational points on moduli spaces, In: Algebraic Number Theory and Related Topics 2007, Res. Inst. Math. Sci. (RIMS) Kôkyûroku Bessatsu B12, Kyoto, 7-29, 2009.

[Grothendieck] Grothendieck, A., Brief an G. Faltings, (German), with an english translation on pp. 285-293, London Math. Soc. Lecture Note Ser., 242, Geometric Galois actions, 1, 49-58, Cambridge Univ. Press, Cambridge, 1997.

[Grothendieck1] Grothendieck, A., Revêtements étales et groupe fondamental, Lecture Notes in Math. 224, Springer, Heidelberg, 1971.

[Hoshi-Mochizuki] Hoshi, Y., Mochizuki, S., On the combinatorial anabelian geometry of nodally nondegenerate outer representations, Hiroshima Math. J. 41 (2011), no.3, 375-342.

[Hoshi-Mochizuki1] Hoshi, Y., Mochizuki, S., Topics surrounding the combinatorial anabelian geometry of hyperbolic curves IV: discretness and sections. Manuscript, available in http://www.kurims.kyoto-u.ac.jp/ motizuki/papers-english.html, (2015). [Lang-Tate] Lang, S., Tate, J., Principal homogeneous spaces over abelian varieties, American Journal of Mathematics, Volume 80, Issue 3, (1958), 659-684.

[Liu] Liu, Q., Algebraic geometry and arithmetic curves, Oxford graduate texts in mathematics 6. Oxford University Press, 2002.

[Mochizuki] Mochizuki, S., The local pro- $p$ anabelian geometry of curves, Invent. Math. 138 (1999), no. 2, 319-423.

[Mochizuki1] Mochizuki, S., The profinite Grothendieck conjecture for closed hyperbolic curves over number fields, J. Math. Sci., Univ. Tokyo 3 (1996), 571-627.

[Mochizuki2] Mochizuki, S., Galois sections in absolute anabelian geometry, Nagoya Math. J. 179 (2005), pp. 17-45.

[Poonen-Voloch] Poonen, B., Voloch, P., The Brauer-Manin obstruction for subvarieties of abelian varieties over function fields, Ann. of Math. (2) 171 (2010), no. 1, 511-532.

[Saïdi] Saïdi, M., The cuspidalisation of sections of arithmetic fundamental groups, Advances in Mathematics, 230 (2012) 1931-1954.

[Saïdi1] Saïdi, M., Revêtements étale abéliens, courants sur les graphes et réduction semi-stable des courbes, Manuscripta Math. 89 (1996), no.2, 245-265.

[Saïdi2] Saïdi, M., On the Grothendieck anabelian section conjecture over finitely generated fields. Talk at the Utah Summer Research Institute on Algebraic Geometry 2015, video talk available on http://www.claymath.org/utah-videos.

[Saïdi-Tamagawa] Saïdi, M., Tamagawa, A., On the arithmetic of abelian varieties, arxiv:1512.00773.

[Serre] Serre, J-P., Lectures on the Mordell-Weil Theorem, Translated and edited by Martin Brown from notes by Michel Waldschmidt, $2^{\text {nd }}$ edition, Aspect of Mathematics, 1990.

[Tamagawa] Tamagawa, A., The Grothendieck conjecture for affine curves, Compositio Math. 109 (1997), no. 2, 135-194.

Mohamed Saïdi 
College of Engineering, Mathematics, and Physical Sciences University of Exeter

Harrison Building

North Park Road

EXETER EX4 4QF

United Kingdom

M.Saidi@exeter.ac.uk 